Research Article

\title{
Stability and Hopf Bifurcation of a Vector-Borne Disease Model with Saturated Infection Rate and Reinfection
}

\author{
Zhixing Hu (D), Shanshan Yin, and Hui Wang (D) \\ School of Mathematics and Physics, University of Science and Technology Beijing, Beijing 100083, China \\ Correspondence should be addressed to Zhixing Hu; huzhixing@ustb.edu.cn
}

Received 28 January 2019; Revised 4 May 2019; Accepted 13 May 2019; Published 9 June 2019

Academic Editor: Luminita Moraru

Copyright (c) 2019 Zhixing Hu et al. This is an open access article distributed under the Creative Commons Attribution License, which permits unrestricted use, distribution, and reproduction in any medium, provided the original work is properly cited.

\begin{abstract}
This paper established a delayed vector-borne disease model with saturated infection rate and cure rate. First of all, according to the basic reproductive number $R_{0}$, we determined the disease-free equilibrium $E_{0}$ and the endemic equilibrium $E_{1}$. Through the analysis of the characteristic equation, we consider the stability of two equilibriums. Furthermore, the effect on the stability of the endemic equilibrium $E_{1}$ by delay was studied, the existence of Hopf bifurcations of this system in $E_{1}$ was analyzed, and the length of delay to preserve stability was estimated. The direction and stability of the Hopf bifurcation were also been determined. Finally, we performed some numerical simulation to illustrate our main results.
\end{abstract}

\section{Introduction}

Malaria is a vector-borne infectious disease [1], caused by parasites. It is popular in 102 countries and regions, especially in some countries in Africa, southeast Asia, and South America. In the 30s of this century, malaria spread throughout the country. Clinical symptoms and signs of this disease, such as typical periodic onset of malaria, secondary anemia, and spleen, can cause serious consequences, including dangerous malaria, malarial kidney disease, and black urine fever.

The main way of transmission of malaria is the bite of an infected female anopheline mosquito. The mosquitoes would also be infected when uninfected mosquitoes bite infected people, and this transmission process has an incubation period [2]. The important feature of malaria is that the recovered immune system may establish immune memory for such antigens. It is this characteristic that greatly reduces the spread of malaria [3, 4]. Immune process is slow and, however, takes years or even decades [5]. As time goes by, the immune system gradually weakens, and at this time, reinfection likely occurs; therefore, considering the function of delay and immune system is necessary in the study of malaria.

For the vector-borne diseases such as malaria, a large number of mathematical models have been created $[2,6,7,8,9]$, most of which consider the local immunity and delay of the spread of malaria in the crowd. Different time delay has been used to describe the latent period in the course of disease transmission [7, 8, 9]. Local stability conditions for the equilibrium of a model with two time delays have been considered by Wan and Cui [8]. The global stability of the equilibrium has been studied for a vectorborne disease model with distributed delay by Cai et al. [10].

Based on the above model, this paper considers a delayed vector-borne model with saturated infection rate and partial immunity to reinfection. We prove that the stability of this system can be changed by time delay and produce Hopf bifurcation, calculating the length of delay to preserve stability. Using the center manifold theorem [11] and norm theory, we determine the stability and bifurcation direction.

\section{Model Formulation}

$N_{1}(t)$ represented as the host population at time $t$ is divided into three subclasses: the susceptible $S(t)$, the infected $I(t)$, and the recovered $R(t) . N_{2}(t)$ represented as the vector population at time $t$ is divided into two subclasses: the susceptible $T(t)$ and the infected $V(t)$. The Hopf bifurcation was determined in a model with direct infection and delay by Wei et al. [9]. The mathematical formulation still needs improvements. We consider an improved model as follows: 


$$
\left\{\begin{array}{l}
\frac{d S}{d t}=\Lambda_{1}-\frac{b \beta_{1} S(t) V(t)}{1+\alpha V(t)}-\mu_{1} S(t), \\
\frac{d I}{d t}=\frac{b \beta_{1} S(t) V(t)}{1+\alpha V(t)}+\sigma b \beta_{1} R(t) V(t)-\left(\mu_{1}+\gamma\right) I(t), \\
\frac{d R}{d t}=\gamma I(t)-\sigma b \beta_{1} R(t) V(t)-\mu_{1} R(t), \\
\frac{d T}{d t}=\Lambda_{2}-b \beta_{2} T(t-\tau) I(t-\tau)-\mu_{2} T(t), \\
\frac{d V}{d t}=b \beta_{2} T(t-\tau) I(t-\tau)-\mu_{2} V(t),
\end{array}\right.
$$

where $\Lambda_{1}$ and $\Lambda_{2}$ represent the recruitment rate of the host population and vector population, respectively. $b$ represents the average number of bites per mosquito per day. The incidence rate $b \beta_{1} S(t) V(t) / 1+\alpha V(t)$ is the number of infections of the susceptible host caused by the infected vector, and $\alpha$ is the inhibitory effect rate caused by the infected vector. $\mu_{1}$ and $\mu_{2}$ represent the death rates of the host population and vector population, respectively. $\beta_{1}$ is the infection rate from vector to human. $\sigma(0 \leq \sigma \leq 1)$ represents the degree of partial protection for recovered people given by a primary infection, where $\sigma=0$ represents complete protection and $\sigma=1$ represents no protection. $\gamma$ is the per capita recovery rate of the infected host population. $\beta_{2}$ represents the infection rate from human to vector. $\tau$ is the time delay, representing the incubation period in the vector population; that is to say, a susceptible vector that bites an infective host at time $t-\tau$ will become infective at time $t$.

The model (1) meets the initial conditions:

$$
\left\{\begin{array}{l}
S(\theta)=\varphi_{1}(\theta) \\
I(\theta)=\varphi_{2}(\theta) \\
R(\theta)=\varphi_{3}(\theta) \\
T(\theta)=\varphi_{4}(\theta) \\
V(\theta)=\varphi_{5}(\theta) \\
\varphi_{i}(\theta) \geq 0, \quad(i=1,2,3,4,5) \\
-\tau \leq \theta \leq 0
\end{array}\right.
$$

where $\left(\varphi_{1}(\theta), \varphi_{2}(\theta), \varphi_{3}(\theta), \varphi_{4}(\theta), \varphi_{5}(\theta)\right) \in C\left([-\tau, 0], R_{+}^{5}\right)$ is the Banach space of continuous functions mapping the interval $[-\tau, 0]$ into $R_{+}^{5}$ with the topology of uniform convergence. The norm is defined as follows:

$$
\|\varphi\|=\sup _{-\tau \leq \theta \leq 0}\left\{\left|\varphi_{1}(\theta)\right|,\left|\varphi_{2}(\theta)\right|,\left|\varphi_{3}(\theta)\right|,\left|\varphi_{4}(\theta)\right|,\left|\varphi_{5}(\theta)\right|\right\} .
$$

Based on the fundamental theory of functional differential equations [12], it is easy to show that the solution of the model (1) with the initial condition (2) is unique and is nonnegative for all $t \geq 0$.

By (1), we know that

$$
N_{k}^{\prime}(t)=\Lambda_{k}-\mu_{k} N_{k}(t), \quad(k=1,2),
$$

and can solve it by using the integrating factor:

$$
N_{k}(t)=N_{k}(0) e^{-\mu_{k} t}+\frac{\Lambda_{k}}{\mu_{k}}\left(1-e^{-\mu_{k} t}\right) .
$$

That is,

$$
\lim _{t \rightarrow \infty} N_{k}(t)=\frac{\Lambda_{k}}{\mu_{k}}, \quad(k=1,2)
$$

Form the limiting theory of differential equation [13], we can draw that model (1) is the equivalent of the following equation:

$$
\left\{\begin{array}{l}
\frac{d S}{d t}=\Lambda_{1}-\frac{b \beta_{1} S(t) V(t)}{1+\alpha V(t)}-\mu_{1} S(t), \\
\frac{d I}{d t}=\frac{b \beta_{1} S(t) V(t)}{1+\alpha V(t)}+\sigma b \beta_{1}\left(\frac{\Lambda_{1}}{\mu_{1}}-S(t)-I(t)\right) \\
\cdot V(t)-\left(\mu_{1}+\gamma\right) I(t), \\
\frac{d V}{d t}=b \beta_{2}\left(\frac{\Lambda_{2}}{\mu_{2}}-V(t-\tau)\right) I(t-\tau)-\mu_{2} V(t) .
\end{array}\right.
$$

Next, the model (7) can be studied in the invariant set:

$$
\Omega=\left\{(S, I, V) \in R_{+}^{3} \mid 0 \leq S+I \leq \frac{\Lambda_{1}}{\mu_{1}}, 0 \leq V \leq \frac{\Lambda_{2}}{\mu_{2}}, S \geq 0, I \geq 0\right\} .
$$

Now, let us consider the existence of equilibrium.

First, it is easy to show that system (7) always has a disease-free equilibrium $E_{0}=\left(S_{0}, I_{0}, V_{0}\right)=\left(\Lambda_{1} / \mu_{1}, 0,0\right)$. The endemic equilibrium $E_{1}=\left(S_{1}, I_{1}, V_{1}\right)$ satisfies the following equation:

$$
\left\{\begin{array}{l}
\Lambda_{1}-\frac{b \beta_{1} S_{1} V_{1}}{1+\alpha V_{1}}-\mu_{1} S_{1}=0 \\
\frac{b \beta_{1} S_{1} V_{1}}{1+\alpha V_{1}}+\sigma b \beta_{1}\left(\frac{\Lambda_{1}}{\mu_{1}}-S_{1}-I_{1}\right) V_{1}-\left(\mu_{1}+\gamma\right) I_{1}=0 \\
b \beta_{2}\left(\frac{\Lambda_{2}}{\mu_{2}}-V_{1}\right) I_{1}-\mu_{2} V_{1}=0
\end{array}\right.
$$

Form (9), we have $S_{1}=\Lambda_{1}\left(1+\alpha V_{1}\right) / b \beta_{1} V_{1}+\mu_{1}\left(1+\alpha V_{1}\right)$ and $I_{1}=\mu_{2} V_{1} / b \beta_{2}\left(\left(\Lambda_{2} / \mu_{2}\right)-V_{1}\right)$, where $V_{1}$ satisfies the following equation:

$$
P_{2} V^{2}+P_{1} V+P_{0}=0
$$

where

$$
\begin{aligned}
P_{2}= & \sigma b \beta_{1}\left[\Lambda_{1} b^{2} \beta_{1} \beta_{2}+\mu_{1} \mu_{2}\left(b \beta_{1}+\alpha \mu_{1}\right)\right], \\
P_{1}= & b^{2} \beta_{1} \beta_{2} \Lambda_{1} \mu_{1}-\sigma b \beta_{1} \Lambda_{1} b^{2} \beta_{1} \beta_{2} \frac{\Lambda_{2}}{\mu_{2}}+\sigma b \beta_{1} \mu_{1}^{2} \mu_{2} \\
& +\mu_{1} \mu_{2}\left(\mu_{1}+\gamma\right)\left(b \beta_{1}+\alpha \mu_{1}\right), \\
P_{0}= & \mu_{1}^{2} \mu_{2}\left(\mu_{1}+\gamma\right)\left(1-R_{0}\right), \\
R_{0}= & \frac{b^{2} \beta_{1} \beta_{2} \Lambda_{1} \Lambda_{2}}{\mu_{1} \mu_{2}^{2}\left(\mu_{1}+\gamma\right)},
\end{aligned}
$$


where $V_{1}=\left(-P_{1}+\sqrt{P_{1}^{2}-4 P_{0} P_{2}}\right) / 2 P_{2}$ and $V_{2}=\left(-P_{1}-\right.$ $\left.\sqrt{P_{1}^{2}-4 P_{0} P_{2}}\right) / 2 P_{2}$ are the two roots of $(10)$ since $\sigma \in[0,1]$, and we have

$$
\begin{aligned}
P_{1} \geq & b^{2} \beta_{1} \beta_{2} \Lambda_{1} \mu_{1}+\sigma b \beta_{1} \mu_{1}^{2} \mu_{2}+\mu_{1} \mu_{2}\left(\mu_{1}+\gamma\right)\left(b \beta_{1}+\alpha \mu_{1}\right) \\
& -b \beta_{1} \Lambda_{1} b^{2} \beta_{1} \beta_{2} \frac{\Lambda_{2}}{\mu_{2}}=b^{2} \beta_{1} \beta_{2} \Lambda_{1} \mu_{1}+\sigma b \beta_{1} \alpha \mu_{1}^{2} \mu_{2} \\
& +b \beta_{1} \mu_{1} \mu_{2}\left(\mu_{1}+\gamma\right)\left(1-R_{0}\right) .
\end{aligned}
$$

Obviously, $P_{2}>0, P_{1}>0$ and $P_{0}>0$ if $R_{0}<1$, and $P_{2}>0$ and $P_{0}<0$ if $R_{0}>1$. From the relationship between roots and coefficients, we know that $V_{1}$ and $V_{2}$ are both negative if $R_{0}<1$ and $V_{1}$ is positive if $R_{0}>1$. According to the above discussion, we can obtain the following theorems.

Theorem 2.1. System (7) has the disease-free equilibrium $E_{0}$ if $R_{0}<1$. System (7) has the disease-free equilibrium $E_{0}$ and an endemic equilibrium $E_{1}$ if $R_{0}>1$.

\section{Stability of Equilibrium and Hopf Bifurcation}

In this section, we study the stability of equilibrium and the existence of Hopf bifurcation of system (7).

The characteristic equation of the linear approximate equation of the system (7) at equilibrium $E=(S, I, V)$ is

$$
\left|\begin{array}{ccc}
\lambda+\frac{b \beta_{1} V}{1+\alpha V}+\mu_{1} & 0 & \frac{b \beta_{1} S}{(1+\alpha V)^{2}} \\
\sigma b \beta_{1} V-\frac{b \beta_{1} V}{1+\alpha V} & \lambda+\sigma b \beta_{1} V+\mu_{1}+\gamma & -\frac{b \beta_{1} S}{(1+\alpha V)^{2}}+Q \\
0 & -b \beta_{2}\left(\frac{\Lambda_{2}}{\mu_{2}}-V\right) e^{-\lambda \tau} & \lambda+\mu_{2}+b \beta_{2} I e^{-\lambda \tau}
\end{array}\right|=0,
$$

where $Q=-\sigma b \beta_{1}\left(\left(\Lambda_{1} / \mu_{1}\right)-S-I\right)$.

3.1. The Local and Global Stability of the Disease-Free Equilibrium. At the disease-free equilibrium $E_{0}$, equation (13) can be expressed as follows:

$$
\left(\lambda+\mu_{1}\right)\left(\lambda^{2}+\left(\mu_{1}+\gamma+\mu_{2}\right) \lambda+\mu_{2}\left(\mu_{1}+\gamma\right)-b^{2} \beta_{1} \beta_{2} \frac{\Lambda_{1} \Lambda_{2}}{\mu_{1} \mu_{2}} e^{-\lambda \tau}\right)=0 .
$$

Obviously, equation (14) has a negative real root $\lambda_{1}=-\mu_{1}$. To discuss the rest of the characteristic roots of (14), we consider the following equation:

$$
\lambda^{2}+\left(\mu_{1}+\gamma+\mu_{2}\right) \lambda+\mu_{2}\left(\mu_{1}+\gamma\right)-b^{2} \beta_{1} \beta_{2} \frac{\Lambda_{1} \Lambda_{2}}{\mu_{1} \mu_{2}} e^{-\lambda \tau}=0 .
$$

When $\tau=0$, equation (15) is equivalent to

$$
\lambda^{2}+\left(\mu_{1}+\gamma+\mu_{2}\right) \lambda+\mu_{2}\left(\mu_{1}+\gamma\right)\left(1-R_{0}\right)=0 \text {. }
$$

By the using Routh-Hurwitz criterion, (16) has two eigenvalues with negative real parts if $R_{0}<1$.

When $\tau>0$, then the roots of (15) can enter the right-half plane in the complex plane by crossing the imaginary axis as the delay $\tau$ increases.

Let $\lambda=\omega i(\omega>0)$ be a purely imaginary root of equation (15), then separating the real and imaginary parts yields

$$
\left\{\begin{array}{l}
-\omega^{2}+\mu_{2}\left(\mu_{1}+\gamma\right)=b^{2} \beta_{1} \beta_{2} \frac{\Lambda_{1} \Lambda_{2}}{\mu_{1} \mu_{2}} \cos \omega \tau, \\
\left(\mu_{2}+\mu_{1}+\gamma\right) \omega=-b^{2} \beta_{1} \beta_{2} \frac{\Lambda_{1} \Lambda_{2}}{\mu_{1} \mu_{2}} \sin \omega \tau .
\end{array}\right.
$$

Squaring and taking the sum of (17) yields

$$
\begin{aligned}
\omega^{4} & +\left(\mu_{2}^{2}+\left(\mu_{1}+\gamma\right)^{2}\right) \omega^{2}+\mu_{2}\left(\mu_{1}+\gamma\right)\left(1-R_{0}\right) \\
& \times\left(\mu_{2}\left(\mu_{1}+\gamma\right)+b^{2} \beta_{1} \beta_{2} \frac{\Lambda_{1} \Lambda_{2}}{\mu_{1} \mu_{2}}\right)=0 .
\end{aligned}
$$

Equation (18) has no roots if $R_{0}<1$. Therefore, we conclude that all eigenvalues of equation (14) have negative real parts.

$$
\begin{aligned}
& \text { If } R_{0}>1 \text {, let } \\
& f(\lambda)=\lambda^{2}+\left(\mu_{1}+\gamma+\mu_{2}\right) \lambda+\mu_{2}\left(\mu_{1}+\gamma\right)-b^{2} \beta_{1} \beta_{2} \frac{\Lambda_{1} \Lambda_{2}}{\mu_{1} \mu_{2}} e^{-\lambda \tau},
\end{aligned}
$$

which implies that

$$
\begin{aligned}
f(0) & =\mu_{2}\left(\mu_{1}+\gamma\right)\left(1-R_{0}\right)<0, \\
\lim _{\lambda \longrightarrow+\infty} f(\lambda) & =+\infty .
\end{aligned}
$$

By the continuity of $f(\lambda)$ and zero point theorem, $f(\lambda)=0$ has at least one positive root. So, the disease-free equilibrium $E_{0}$ is unstable. Based on the results, we can draw the conclusion.

Theorem 3.1. For any $\tau$, the virus-free equilibrium $E_{0}$ of the system (7) is locally asymptotically stable if $R_{0}<1$, and it is unstable if $R_{0}>1$.

In fact, using a similar approach to the literature [14], we can know that $E_{0}$ is globally asymptotically stable if $R_{0}<1$. A detailed proof is given below.

For a continuous and bounded function $f(t)$, we define

$$
\begin{aligned}
& f^{\infty} \triangleq \lim _{t \longrightarrow \infty} \sup f(t), \\
& f_{\infty} \triangleq \lim _{t \longrightarrow \infty} \inf f(t) .
\end{aligned}
$$

For system (7), any solution with the initial conditions is $(S(t), I(t), V(t))$, and we have

$$
\begin{aligned}
& 0 \leq S_{\infty} \leq S^{\infty} \leq \infty \\
& 0 \leq I_{\infty} \leq I^{\infty} \leq \infty \\
& 0 \leq V_{\infty} \leq V^{\infty} \leq \infty
\end{aligned}
$$


By the fluctuation lemma [15], we know that there is a sequence $\left\{t_{n}\right\}$; when $t_{n} \longrightarrow \infty$, we have $S\left(t_{n}\right) \longrightarrow S^{\infty}$ and $S^{\prime}\left(t_{n}\right) \longrightarrow 0(n \longrightarrow \infty)$. Substituting $t_{n}$ into the first equation of (7) yields

$$
S^{\prime}\left(t_{n}\right)=\Lambda_{1}-\frac{b \beta_{1} S\left(t_{n}\right) V\left(t_{n}\right)}{1+\alpha V\left(t_{n}\right)}-\mu_{1} S\left(t_{n}\right) \leq \Lambda_{1}-\mu_{1} S\left(t_{n}\right)
$$

Let us take the limits on both sides:

$$
\lim _{n \longrightarrow \infty} S^{\prime}\left(t_{n}\right) \leq \Lambda_{1}-\mu_{1} \lim _{n \longrightarrow \infty} S\left(t_{n}\right) \text {, i.e., } \mu_{1} S^{\infty} \leq \Lambda_{1} \text {. }
$$

Similarly,

$$
\begin{aligned}
\left(\mu_{1}+\gamma\right) I^{\infty} & \leq(1-\sigma) b \beta_{1} S^{\infty} V^{\infty}+\sigma b \beta_{1} \frac{\Lambda_{1}}{\mu_{1}} V^{\infty}, \\
\mu_{2} V^{\infty} & \leq b \beta_{2} \frac{\Lambda_{2}}{\mu_{2}} I^{\infty} .
\end{aligned}
$$

Combining (24) and (25), we know that

$$
\mu_{2} V^{\infty} \leq b^{2} \beta_{1} \beta_{2} \frac{\Lambda_{1} \Lambda_{2}}{\mu_{1} \mu_{2}\left(\mu_{1}+\gamma\right)} V^{\infty} .
$$

Since $V^{\infty}$ is the supremum of the function $V(t), V^{\infty} \geq 0$. If $V^{\infty}>0$, by using (26), $\mu_{2} \leq b^{2} \beta_{1} \beta_{2}\left(\Lambda_{1} \Lambda_{2} / \mu_{1} \mu_{2}\left(\mu_{1}+\gamma\right)\right)$, which contradicts $R_{0}<1$. That is to say, $V^{\infty}=0$, which implies that $\lim _{t \rightarrow \infty} V(t)=0$. In the same way by using (25), we have $\lim _{t \longrightarrow \infty} I(t)=0$. According to the limit theorem [13], we have $\lim _{t \rightarrow \infty} S(t)=\Lambda_{1} / \mu_{1}$. Combined with the local asymptotic stability of $E_{0}$, we can get the following theorem:

Theorem 3.2. For any $\tau$, the virus-free equilibrium $E_{0}$ of system (7) is globally asymptotically stable if $R_{0}<1$.

3.2. The Local Stability of the Endemic Equilibrium. From (13), the characteristic equation of linear approximate equation of the system (7) at the endemic equilibrium $E_{1}$ is

$$
\lambda^{3}+A_{2} \lambda^{2}+A_{1} \lambda+A_{0}+\left(B_{2} \lambda^{2}+B_{1} \lambda+B_{0}\right) e^{-\lambda \tau}=0,
$$

where

$$
\begin{aligned}
A_{2}= & \frac{b \beta_{1} V_{1}}{1+\alpha V_{1}}+2 \mu_{1}+\sigma b \beta_{1} V_{1}+\gamma+\mu_{2}, \\
A_{1}= & \left(\frac{b \beta_{1} V_{1}}{1+\alpha V_{1}}+\mu_{1}\right)\left(\sigma b \beta_{1} V_{1}+\mu_{1}+\gamma+\mu_{2}\right) \\
& +\left(\sigma b \beta_{1} V_{1}+\mu_{1}+\gamma\right) \mu_{2}, \\
A_{0}= & \left(\frac{b \beta_{1} V_{1}}{1+\alpha V_{1}}+\mu_{1}\right)\left(\sigma b \beta_{1} V_{1}+\mu_{1}+\gamma\right) \mu_{2}, \\
B_{2}= & b \beta_{2} I_{1}, \\
B_{1}= & b \beta_{2} I_{1}\left(\frac{b \beta_{1} V_{1}}{1+\alpha V_{1}}+\sigma b \beta_{1} V_{1}+2 \mu_{1}+\gamma\right) \\
& -\frac{b \beta_{1} S_{1}}{1+\alpha V_{1}}\left(\frac{1}{1+\alpha V_{1}}-1\right) \mu_{2} \frac{V_{1}}{I_{1}}-\mu_{2}\left(\mu_{1}+\gamma\right), \\
B_{0}= & b \beta_{2} I_{1}\left(\frac{b \beta_{1} V_{1}}{1+\alpha V_{1}}+\mu_{1}\right)\left(\sigma b \beta_{1} V_{1}+\mu_{1}+\gamma\right) \\
& -\frac{b \beta_{1} S_{1}}{1+\alpha V_{1}}\left(\frac{b \beta_{1} V_{1}}{1+\alpha V_{1}}+\mu_{1}\right)\left(\frac{1}{1+\alpha V_{1}}-1\right) \\
& \times \mu_{2} \frac{V_{1}}{I_{1}}-\left(\frac{b \beta_{1} V_{1}}{1+\alpha V_{1}}+\mu_{1}\right) \mu_{2}\left(\mu_{1}+\gamma\right) \\
& \left(\frac{b \beta_{1} V_{1}}{1+\alpha V_{1}}-\sigma b \beta_{1} V_{1}\right) \frac{b \beta_{1} S_{1}}{\left(1+\alpha V_{1}\right)^{2}} \mu_{2} \frac{V_{1}}{I_{1}} .
\end{aligned}
$$

When $\tau=0$, equation (27) is equivalent to

$$
\lambda^{3}+H_{2} \lambda^{2}+H_{1} \lambda+H_{0}=0,
$$

where

$$
\begin{aligned}
H_{2}= & A_{2}+B_{2}=\frac{b \beta_{1} V_{1}}{1+\alpha V_{1}}+b \beta_{2} I_{1}+2 \mu_{1}+\mu_{2}+\sigma b \beta_{1} V_{1}+\gamma>0, \\
H_{1}= & A_{1}+B_{1}=\left(\frac{b \beta_{1} V_{1}}{1+\alpha V_{1}}+\mu_{1}\right)\left(\sigma b \beta_{1} V_{1}+\mu_{1}+\gamma+\mu_{2}\right)+\sigma b \beta_{1} V_{1} \mu_{2}+b \beta_{2} I_{1}\left(\frac{b \beta_{1} V_{1}}{1+\alpha V_{1}}+\sigma b \beta_{1} V_{1}+2 \mu_{1}+\gamma\right) \\
& +\frac{b \beta_{1} S_{1}}{1+\alpha V_{1}}\left(1-\frac{1}{1+\alpha V_{1}}\right) \mu_{2} \frac{V_{1}}{I_{1}}>0 \\
H_{0}= & A_{0}+B_{0}=\left(\frac{b \beta_{1} V_{1}}{1+\alpha V_{1}}+\mu_{1}\right)\left[\left(\sigma b \beta_{1} V_{1}+\mu_{1}+\gamma\right) b \beta_{2} I_{1}+\sigma b \beta_{1} V_{1} \mu_{2}\right]+(1-\sigma) b \beta_{1} V_{1} \frac{b \beta_{1} S_{1}}{\left(1+\alpha V_{1}\right)^{2}} \frac{\mu_{2} V_{1}}{I_{1}} \\
& +\mu_{1} \frac{b \beta_{1} S_{1}}{1+\alpha V_{1}}\left(1-\frac{1}{1+\alpha V_{1}}\right) \frac{\mu_{2} V_{1}}{I_{1}}>0 .
\end{aligned}
$$


Notice that

$$
\begin{aligned}
H_{2} H_{1}-H_{0}= & \left(\frac{b \beta_{1} V_{1}}{1+\alpha V_{1}}+b \beta_{2} I_{1}+2 \mu_{1}+\mu_{2}+\sigma b \beta_{1} V_{1}+\gamma\right)\left[\left(\frac{b \beta_{1} V_{1}}{1+\alpha V_{1}}+\mu_{1}\right) \times\left(\sigma b \beta_{1} V_{1}+\mu_{1}+\gamma+\mu_{2}\right)+\sigma b \beta_{1} V_{1} \mu_{2}\right. \\
& \left.+b \beta_{2} I_{1}\left(\frac{b \beta_{1} V_{1}}{1+\alpha V_{1}}+\sigma b \beta_{1} V_{1}+2 \mu_{1}+\gamma\right)+\frac{b \beta_{1} S_{1}}{1+\alpha V_{1}}\left(1-\frac{1}{1+\alpha V_{1}}\right) \mu_{2} \frac{V_{1}}{I_{1}}\right] \\
& -\left(\frac{b \beta_{1} V_{1}}{1+\alpha V_{1}}+\mu_{1}\right)\left[\left(\sigma b \beta_{1} V_{1}+\mu_{1}+\gamma\right) b \beta_{2} I_{1}+\sigma b \beta_{1} V_{1} \mu_{2}\right]-(1-\sigma) b \beta_{1} V_{1} \frac{b \beta_{1} S_{1}}{\left(1+\alpha V_{1}\right)^{2}} \frac{\mu_{2} V_{1}}{I_{1}} \\
& -\mu_{1} \frac{b \beta_{1} S_{1}}{1+\alpha V_{1}}\left(1-\frac{1}{1+\alpha V_{1}}\right) \frac{\mu_{2} V_{1}}{I_{1}} \geq\left(\frac{b \beta_{1} V_{1}}{1+\alpha V_{1}}+\mu_{1}+\mu_{2}\right)\left[\frac{b \beta_{1} V_{1}}{1+\alpha V_{1}}\left(\gamma+\mu_{1}\right)+\sigma b \beta_{1} V_{1} \mu_{2}+b \beta_{2} I_{1}\right. \\
& \left.\times\left(\sigma b \beta_{1} V_{1}+\mu_{1}+\gamma\right)\right]+\frac{b \beta_{1} S_{1}}{1+\alpha V_{1}}\left(1-\frac{1}{1+\alpha V_{1}}\right) \mu_{2} \frac{V_{1}}{I_{1}}\left(\frac{b \beta_{1} V_{1}}{1+\alpha V_{1}}+\mu_{1}\right)-\left(\frac{b \beta_{1} V_{1}}{1+\alpha V_{1}}+\mu_{1}\right) \\
& \cdot\left[\left(\sigma b \beta_{1} V_{1}+\mu_{1}+\gamma\right) b \beta_{2} I_{1}+\sigma b \beta_{1} V_{1} \mu_{2}\right]-(1-\sigma) b \beta_{1} V_{1} \frac{b \beta_{1} S_{1}}{\left(1+\alpha V_{1}\right)^{2}} \frac{\mu_{2} V_{1}}{I_{1}}-\mu_{1} \frac{b \beta_{1} S_{1}}{1+\alpha V_{1}}\left(1-\frac{1}{1+\alpha V_{1}}\right) \frac{\mu_{2} V_{1}}{I_{1}} \\
& \geq \frac{b \beta_{1} V_{1}}{1+\alpha V_{1}} \mu_{2}\left(\gamma+\mu_{1}\right)-\frac{b \beta_{1} S_{1}}{\left(1+\alpha V_{1}\right)^{2}} \frac{\mu_{2} V_{1}}{I_{1}} \frac{b \beta_{1} V_{1}}{1+\alpha V_{1}}+\sigma b \beta_{1} V_{1} \frac{b \beta_{1} S_{1}}{\left(1+\alpha V_{1}\right)^{2}} \frac{\mu_{2} V_{1}}{I_{1}}, \\
\frac{V_{1}}{I_{1}}= & \frac{\mu_{1}+\gamma}{\left(b \beta_{1} S_{1} / 1+\alpha V_{1}\right)+\sigma b \beta_{1}\left(\left(\Lambda_{1} / \mu_{1}\right)-S_{1}-I_{1}\right)} \leq \frac{\left.\mu \beta_{1} S_{1} /\left(1+\alpha V_{1}\right)\right)}{\mu_{1}+\gamma} \frac{\left(\mu_{1}+\gamma\right)\left(1+\alpha V_{1}\right)}{b \beta_{1} S_{1}} .
\end{aligned}
$$

It follows that

$$
\begin{aligned}
H_{2} H_{1}-H_{0} \geq & \frac{b \beta_{1} V_{1}}{1+\alpha V_{1}} \mu_{2}\left(\gamma+\mu_{1}\right)-\frac{b \beta_{1} S_{1}}{\left(1+\alpha V_{1}\right)^{2}} \\
& \cdot \frac{b \beta_{1} V_{1}}{1+\alpha V_{1}} \mu_{2} \frac{\left(\mu_{1}+\gamma\right)\left(1+\alpha V_{1}\right)}{b \beta_{1} S_{1}} \\
& =\frac{b \beta_{1} V_{1}}{1+\alpha V_{1}} \mu_{2}\left(\gamma+\mu_{1}\right)-\frac{b \beta_{1} V_{1}}{\left(1+\alpha V_{1}\right)^{2}}\left(\gamma+\mu_{1}\right) \mu_{2} \\
& =\frac{b \beta_{1} V_{1}}{1+\alpha V_{1}} \mu_{2}\left(\gamma+\mu_{1}\right)\left(1-\frac{1}{1+\alpha V_{1}}\right)>0 .
\end{aligned}
$$

By using the Routh-Hurwitz criterion, equation (29) only has eigenvalues with negative real parts if $R_{0}>1$. We can obtain the following theorem:

Theorem 3.3. For $\tau=0$, the endemic equilibrium $E_{1}$ of system (7) is locally asymptotically stable if $R_{0}>1$.
3.3. Hopf Bifurcation. In this subsection, we devote to investigating the stability of the endemic equilibrium and the existence of Hopf bifurcation.

Let $\lambda=\omega i(\omega>0)$ be the root of equation (27), substituting it into equation (27) and separating the real and imaginary parts; we can obtain the following equation:

$$
\left\{\begin{array}{l}
A_{2} \omega^{2}-A_{0}=\left(B_{0}-B_{2} \omega^{2}\right) \cos \omega \tau+B_{1} \omega \sin \omega \tau, \\
\omega^{3}-A_{1} \omega=B_{1} \omega \cos \omega \tau-\left(B_{0}-B_{2} \omega^{2}\right) \sin \omega \tau .
\end{array}\right.
$$

Squaring and taking the sum of (34) yields

$$
\omega^{6}+p_{2} \omega^{4}+p_{1} \omega^{2}+p_{0}=0
$$

where

$$
\begin{aligned}
& p_{2}=A_{2}^{2}-2 A_{1}-B_{2}^{2}, \\
& p_{1}=A_{1}^{2}-2 A_{0} A_{2}+2 B_{0} B_{2}-B_{1}^{2}, \\
& p_{0}=A_{0}^{2}-B_{0}^{2} .
\end{aligned}
$$

Let $x=\omega^{2}$, then equation (35) is equivalent to

$$
f(x) \triangleq x^{3}+p_{2} x^{2}+p_{1} x+p_{0}=0,
$$

then $f^{\prime}(x)=3 x^{2}+2 p_{2} x+p_{1}$. The two roots of equation $3 x^{2}+2 p_{2} x+p_{1}=0$ are 


$$
\begin{aligned}
x^{*} & =\frac{-p_{2}+\sqrt{p_{2}^{2}-3 p_{1}}}{3}, \\
x^{* *} & =\frac{-p_{2}-\sqrt{p_{2}^{2}-3 p_{1}}}{3} .
\end{aligned}
$$

According to [16], the condition that equation (37) has positive roots is as follows:

Lemma 3.4. For equation (37),

(i) If $p_{0}<0$, then equation (37) has at least one positive root

(ii) If $p_{0} \geq 0$ and $p_{2}^{2} \leq 3 p_{1}$, then equation (37) has no positive root (iii) If $p_{0} \geq 0$ and $p_{2}^{2}>3 p_{1}$, then equtaion (37) has positive roots if and only if $x^{*}>0$ and $f\left(x^{*}\right) \leq 0$

Based on Lemma 3.4, we concluded that if (ii) is set up, then the stability of $E_{1}$ will not change when $\tau$ increases. If equation (37) has a positive root, then the stability of $E_{1}$ may change with the change in $\tau$.

Suppose that equation (37) has three positive roots, written as $x_{1}, x_{2}$, and $x_{3}$. Then, equation (35) has positive roots $\omega_{k}=\sqrt{x_{k}}(k=1,2,3)$. By using (34),

$$
\cos \omega \tau=\frac{\left(B_{0}-B_{2} \omega^{2}\right)\left(A_{2} \omega^{2}-A_{0}\right)+B_{1} \omega\left(\omega^{3}-A_{1} \omega\right)}{\left(B_{0}-B_{2} \omega^{2}\right)^{2}+\left(B_{1} \omega\right)^{2}} .
$$

Define

$$
\tau_{k}^{(j)}=\frac{1}{\omega_{k}}\left\{\arccos \frac{\left(B_{0}-B_{2} \omega_{k}^{2}\right)\left(A_{2} \omega_{k}^{2}-A_{0}\right)+B_{1} \omega_{k}\left(\omega_{k}^{3}-A_{1} \omega_{k}\right)}{\left(B_{0}-B_{2} \omega_{k}^{2}\right)^{2}+\left(B_{1} \omega_{k}\right)^{2}}+2 j \pi\right\},
$$

where $k=1,2,3, j=0,1, \ldots$ Obviously, $\pm \omega_{k} i$ is a pair of pure virtual root of equation (27).

Let

$$
\begin{aligned}
\tau^{*} & =\tau_{k_{0}}^{(0)}=\min _{k \in\{1,2,3\}}\left\{\tau_{k}^{(0)}\right\}, \\
\omega^{*} & =\omega_{k_{0}}
\end{aligned}
$$

It follows that $\lambda(\tau)=\xi_{0}(\tau)+i \omega(\tau)$ is the root of then equation (35) satisfying $\xi_{0}\left(\tau^{*}\right)=0$ and $\omega\left(\tau^{*}\right)=\omega^{*}$.
Next, we verify the transversal condition. Differentiating the two sides of equation (35) with respect to $\tau$, we have

$$
\begin{aligned}
\left(\frac{d \lambda}{d \tau}\right)^{-1}= & -\frac{3 \lambda^{2}+2 A_{2} \lambda+A_{1}}{\lambda\left(\lambda^{3}+A_{2} \lambda^{2}+A_{1} \lambda+A_{0}\right)} \\
& +\frac{2 \lambda B_{2}+B_{0}}{\lambda\left(B_{2} \lambda^{2}+B_{1} \lambda+B_{0}\right)}-\frac{\tau}{\lambda}
\end{aligned}
$$

Thus,

$$
\begin{aligned}
\operatorname{sign}\left\{\left.\frac{d \operatorname{Re}(\lambda)}{d \tau}\right|_{\tau=\tau^{*}}\right\} & =\operatorname{sign}\left\{\operatorname{Re}\left[\left.\left(\frac{d \lambda}{d \tau}\right)^{-1}\right|_{\tau=\tau^{*}}\right]\right\} \\
& =\operatorname{sign}\left\{f^{\prime}\left(\omega^{* 2}\right)\right\} .
\end{aligned}
$$

If $f^{\prime}\left(\omega^{* 2}\right)>0$, the transversal condition is satisfied. Therefore, according to the above discussion and the Hopf bifurcation theorem of the differential equations [12], we can get the following result:
Theorem 3.5.

(i) If $p_{0} \geq 0$ and $\Delta=p_{2}^{2}-3 p_{1} \leq 0$, then the endemic equilibrium $E_{1}$ is locally asymptotically stable for all $\tau>0$.

(ii) If $p_{0}<0$ or $p_{0} \geq 0, \Delta>0, x^{*}>0$, and $f\left(x^{*}\right) \leq 0$, then the endemic equilibrium $E_{1}$ is locally asymptotically stable for $0<\tau<\tau^{*}$, and if $f^{\prime}\left(\omega^{*^{2}}\right) \neq 0$, the system (7) undergoes a Hopf bifurcation at $E_{1}$ when $\tau=\tau^{*}$, where 


$$
\tau^{*}=\frac{1}{\omega^{*}}\left\{\arccos \frac{\left(B_{0}-B_{2} \omega^{*^{2}}\right)\left(A_{2} \omega^{*^{2}}-A_{0}\right)+B_{1} \omega^{*}\left(\omega^{*^{3}}-A_{1} \omega^{*}\right)}{\left(B_{0}-B_{2} \omega^{*^{2}}\right)^{2}+\left(B_{1} \omega^{*}\right)^{2}}\right\} .
$$

\section{Estimation of the Length of Delay to Preserve Stability}

In this section, we use a Nyquist criterion [17] to calculate the length of delay to preserve stability.

Consider the system (7) and the space of the real continuous functions that is defined in $[-\tau,+\infty]$ and satisfied the initial conditions (2) in the interval $[-\tau, 0]$. Define

$$
\begin{aligned}
S(t) & =S_{1}+X(t), \\
I(t) & =I_{1}+Y(t), \\
V(t) & =V_{1}+Z(t) .
\end{aligned}
$$

Linearization system (7) at the endemic equilibrium $E_{1}$ is expressed as follows:

$$
\left\{\begin{array}{l}
\frac{d X(t)}{d t}=-\left(\frac{b \beta_{1} V_{1}}{1+\alpha V_{1}}+\mu_{1}\right) X(t)-\frac{b \beta_{1} S_{1}}{\left(1+\alpha V_{1}\right)^{2}} Z(t), \\
\frac{d Y(t)}{d t}=\left(\frac{b \beta_{1} V_{1}}{1+\alpha V_{1}}-\sigma b \beta_{1} V_{1}\right) X(t)-\left(\sigma b \beta_{1} V_{1}+\mu_{1}+\gamma\right) Y(t) \\
+\left[\frac{b \beta_{1} S_{1}}{\left(1+\alpha V_{1}\right)^{2}}+\sigma b \beta_{1}\left(\frac{\Lambda_{1}}{\mu_{1}}-S_{1}-I_{1}\right)\right] Z(t), \\
\frac{d Z(t)}{d t}=-\mu_{2} Z(t)+b \beta_{2}\left(\frac{\Lambda_{2}}{\mu_{2}}-V_{1}\right) Y(t-\tau)-b \beta_{2} I_{1} Z(t-\tau) .
\end{array}\right.
$$

By taking the Laplace transformation for (47), we can obtain

$$
\left\{\begin{array}{l}
S L[X]-X(0)=-\left(\frac{b \beta_{1} V_{1}}{1+\alpha V_{1}}+\mu_{1}\right) L[X]-\frac{b \beta_{1} S_{1}}{\left(1+\alpha V_{1}\right)^{2}} L[Z], \\
S L[Y]-Y(0)=\left(\frac{b \beta_{1} V_{1}}{1+\alpha V_{1}}-\sigma b \beta_{1} V_{1}\right) L[X]-\left(\sigma b \beta_{1} V_{1}+\mu_{1}+\gamma\right) L[Y]+\left[\frac{b \beta_{1} S_{1}}{\left(1+\alpha V_{1}\right)^{2}}+\sigma b \beta_{1}\left(\frac{\Lambda_{1}}{\mu_{1}}-S_{1}-I_{1}\right)\right] L[Z], \\
S L[Z]-Z(0)=-\mu_{2} L[Z]+b \beta_{2}\left(\frac{\Lambda_{2}}{\mu_{2}}-V_{1}\right) L\left[Y_{\tau}\right]-b \beta_{2} I_{1} L\left[Z_{\tau}\right],
\end{array}\right.
$$

where

$$
\begin{aligned}
L\left[Y_{\tau}\right]= & \int_{0}^{\infty} e^{-s t} Y(t-\tau) d t=\int_{0}^{\tau} e^{-s t} Y(t-\tau) d t \\
& +\int_{\tau}^{\infty} e^{-s t} Y(t-\tau) d t .
\end{aligned}
$$

Let $t=t_{1}+\tau$, then

$$
\begin{aligned}
L\left[Y_{\tau}\right] & =\int_{-\tau}^{0} e^{-s\left(t_{1}+\tau\right)} Y\left(t_{1}\right) d t_{1}+\int_{0}^{\infty} e^{-s\left(t_{1}+\tau\right)} Y\left(t_{1}\right) d t_{1} \\
& =M_{1} e^{-s \tau}+e^{-s \tau} L[Y]
\end{aligned}
$$

where $M_{1}=\int_{-\tau}^{0} e^{-s t} Y(t) d t$.
Similarly,

$$
\begin{aligned}
L\left[Y_{\tau}\right] & =\int_{-\tau}^{0} e^{-s\left(t_{1}+\tau\right)} Y\left(t_{1}\right) d t_{1}+\int_{0}^{\infty} e^{-s\left(t_{1}+\tau\right)} Y\left(t_{1}\right) d t_{1} \\
& =M_{1} e^{-s \tau}+e^{-s \tau} L[Y]
\end{aligned}
$$

where $M_{2}=\int_{-\tau}^{0} e^{-s t} Z(t) d t$.

Thus, (48) can be written as

$$
(A-S I)\left(\begin{array}{c}
L[X] \\
L[Y] \\
L[Z]
\end{array}\right)=B,
$$

where 


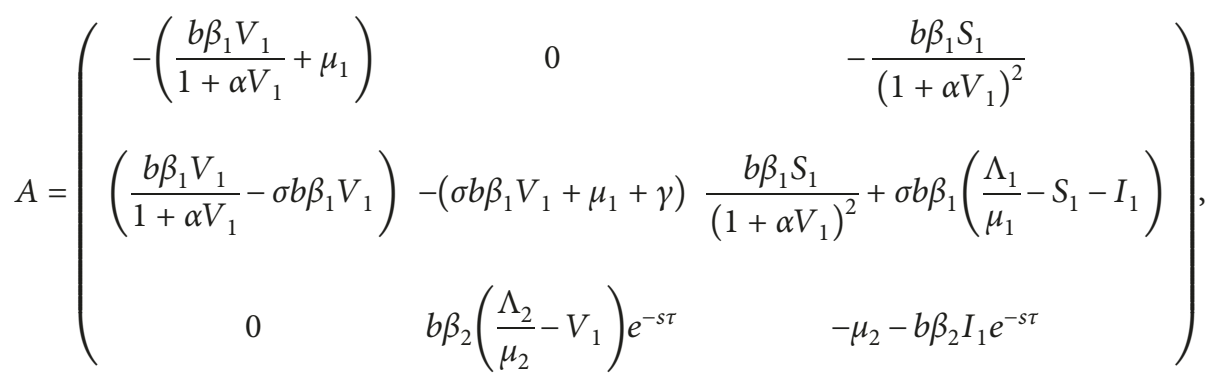

$$
\begin{aligned}
& B=\left(\begin{array}{c}
-X(0) \\
-Y(0) \\
-Z(0)+\left[b \beta_{2} I_{1} M_{2}-b \beta_{2}\left(\frac{\Lambda_{2}}{\mu_{2}}-V_{1}\right) M_{1}\right] e^{-s \tau}
\end{array}\right) .
\end{aligned}
$$

The inverse Laplace transformation of $L[X(t)], L[Y(t)]$, and $L[Z(t)]$ will have terms which exponentially increase with time if $L[X(t)], L[Y(t)]$, and $L[Z(t)]$ have poles with positive real parts. Thus, $E_{1}$ is locally asymptotically stable if and only if all the poles of $L[X(t)], L[Y(t)]$, and $L[Z(t)]$ have negative real parts.

By the method of [17] and the Nyquist criterion, the local asymptotic stability of $E_{1}$ needs to satisfy the following two conditions:

$$
\begin{aligned}
& \operatorname{Re} F\left(i \mu_{0}\right)=0, \\
& \operatorname{Im} F\left(i \mu_{0}\right)>0,
\end{aligned}
$$

where

$$
F(s)=s^{3}+A_{2} s^{2}+A_{1} s+A_{0}+\left(B_{2} s^{2}+B_{1} s+B_{0}\right) e^{-s \tau},
$$

where $\mu_{0}$ is the smallest positive root of (54). Thus (54) and (55) can be written as

$$
\begin{array}{r}
A_{2} \mu_{0}^{2}-A_{0}=\left(B_{0}-B_{2} \mu_{0}^{2}\right) \cos \mu_{0} \tau+B_{1} \mu_{0} \sin \mu_{0} \tau, \\
-\mu_{0}^{3}+A_{1} \mu_{0}>\left(B_{0}-B_{2} \mu_{0}^{2}\right) \sin \mu_{0} \tau-B_{1} \mu_{0} \cos \mu_{0} \tau .
\end{array}
$$

In order to estimate the length of delay to preserve stability, under the premise of ensuring stability, the following conditions need to be satisfied:

$$
\begin{aligned}
& A_{2} \mu^{2}-A_{0}=\left(B_{0}-B_{2} \mu^{2}\right) \cos \mu \tau+B_{1} \mu \sin \mu \tau, \\
& -\mu^{3}+A_{1} \mu>\left(B_{0}-B_{2} \mu^{2}\right) \sin \mu \tau-B_{1} \mu \cos \mu \tau .
\end{aligned}
$$

If (58) and (59) are satisfied simultaneously, they are sufficient conditions to guarantee stability. Our aim is to find an upper bound $\mu_{+}$to $\mu_{0}$ independent of $\tau$ and then to estimate $\tau$ so that (59) holds true for all values of $0 \leq \mu \leq \mu_{+}$ and in particular at $\mu=\mu_{0}$.

Since $|\cos \mu \tau| \leq 1$ and $|\sin \mu \tau| \leq 1$, from equation (58), we have

$$
A_{2} \mu^{2} \leq\left|B_{0}\right|+B_{2} \mu^{2}+\left|B_{1}\right| \mu+A_{0} .
$$

Let

$$
\mu_{+}=\frac{\left|B_{1}\right|+\sqrt{B_{1}^{2}+4\left(A_{2}-B_{2}\right)\left(A_{0}+\left|B_{0}\right|\right)}}{2\left(A_{2}-B_{2}\right)},
$$

obviously $\mu_{+}$meets (60) and $\mu_{+} \geq \mu_{0}$.

From equation (59), we obtain

$$
\mu^{2}<B_{1} \cos \mu \tau+\left(B_{2} \mu-\frac{B_{0}}{\mu}\right) \sin \mu \tau+A_{1} .
$$

Since $E_{1}$ is locally asymptotically stable for $\tau=0$, the inequality (62) will continue to hold for sufficiently small $\tau$ and $\mu=\mu_{0}$.

On the basis of (58) and (62), we have

$$
\begin{gathered}
\left(B_{1} \mu-A_{2} B_{2} \mu+\frac{A_{2} B_{0}}{\mu}\right) \sin \mu \tau+\left(B_{2} \mu^{2}+A_{2} B_{1}-B_{0}\right) \\
\cdot(1-\cos \mu \tau)<B_{2} \mu^{2}+A_{1} A_{2}+A_{2} B_{1}-A_{0}-B_{0} .
\end{gathered}
$$

Note the left-hand side of $(63)$ is $\Phi(\tau, \mu)$ and the righthand side is $\rho$. By using inequality $\sin \mu \tau \leq \mu \tau$ and $1-\cos \mu \tau=2 \sin ^{2}(\mu \tau / 2) \leq\left(\mu^{2} \tau^{2} / 2\right)$, we can obtain

$$
\begin{aligned}
\Phi(\tau, \mu) \leq|\Phi(\tau, \mu)| \leq & \left(\left|B_{1}-A_{2} B_{2}\right| \mu+\left|\frac{A_{2} B_{0}}{\mu}\right|\right) \mu \tau \\
& +\left|B_{2} \mu^{2}+A_{2} B_{1}-B_{0}\right| \frac{\mu^{2} \tau^{2}}{2} \\
& =\left(\left|B_{1}-A_{2} B_{2}\right| \mu^{2}+\left|A_{2} B_{0}\right|\right) \tau \\
& +\left|B_{2} \mu^{2}+A_{2} B_{1}-B_{0}\right| \frac{\mu^{2} \tau^{2}}{2} .
\end{aligned}
$$

Note the right-hand side of (64) is $\varphi(\tau, \mu)$. Clearly, $\Phi(\tau, \mu) \leq \varphi(\tau, \mu) \leq \varphi\left(\tau, \mu_{+}\right)$when $\mu \in\left[0, \mu_{+}\right]$. Thus, if $\varphi\left(\tau, \mu_{+}\right) \leq \rho \leq K_{3}$, we have $\Phi\left(\tau, \mu_{+}\right) \leq \rho \leq K_{3}$. Let $\tau^{*}$ be the positive root of $\varphi\left(\tau, \mu_{+}\right)=K_{3}$, that is,

$$
\tau^{*}=\frac{1}{2 K_{1}}\left(-K_{2}+\sqrt{K_{2}^{2}+4 K_{1} K_{3}}\right) \text {, }
$$


where

$$
\begin{aligned}
& K_{1}=\frac{\left|B_{2} \mu_{+}^{2}+A_{2} B_{1}-B_{0}\right| \mu_{+}^{2}}{2}, \\
& K_{2}=\left|B_{1}-A_{2} B_{2}\right| \mu_{+}^{2}+\left|A_{2} B_{0}\right|, \\
& K_{3}=B_{2} \mu_{+}^{2}+A_{1} A_{2}+A_{2} B_{1}-A_{0}-B_{0} .
\end{aligned}
$$

Summarizing the above discussions, we have the following theorem.

Theorem 4.1. If $0<\tau<\tau^{*}$, then the Nyquist criterion holds true and $\tau^{*}$ estimates the maximum length of the delay preserving the stability, where $\tau^{*}$ satisfies (65).

\section{Direction and Stability of the Hopf Bifurcation}

We have obtained the conditions under which the Hopf bifurcation occurs at $E_{1}$ of the system (7). This section will use the normal form theory and the center manifold theory to give the direction of the Hopf bifurcation and the stability of the bifurcating periodic solutions of system (7). We suppose that system (7) undergoes Hopf bifurcation at $E_{1}$ for $\tau=\tilde{\tau}\left(\tilde{\tau}=\tau_{k}^{(j)}\right)$. Let $\pm i \omega$ be a pair of conjugate pure virtual roots at $E_{1}$ when $\tau=\tilde{\tau}$.

Define

$$
\begin{aligned}
x_{1}(t) & =S(\tau t)-S_{1}, \\
x_{2}(t) & =I(\tau t)-I_{1}, \\
x_{3}(t) & =V(\tau t)-V_{1}, \\
\tau & =\tilde{\tau}+\mu .
\end{aligned}
$$

Thus, system (7) is equivalent to the following functional differential equation in $C=C\left([-\tau, 0], R^{3}\right)$.

$$
\frac{d x}{d t}=L_{\mu}\left(x_{t}\right)+f\left(\mu, x_{t}\right) \text {, }
$$

where $x(t)=\left(x_{1}(t), x_{2}(t), x_{3}(t)\right)^{\mathrm{T}} \in R^{3}$. And $L_{\mu}: C \longrightarrow R^{3}$ and $f: R \times C \longrightarrow R^{3}$ satisfy

$$
\begin{aligned}
L_{\mu}(\phi) & =(\tilde{\tau}+\mu) B_{1} \phi(0)+(\tilde{\tau}+\mu) B_{2} \phi(-1), \\
f(\mu, \phi) & =(\widetilde{\tau}+\mu)\left(f_{1}, f_{2}, f_{3}\right)^{\mathrm{T}},
\end{aligned}
$$

where

$$
\begin{aligned}
& B_{1}=\left(\begin{array}{ccc}
-\left(\frac{b \beta_{1} V_{1}}{1+\alpha V_{1}}+\mu_{1}\right) & 0 & -\frac{b \beta_{1} S_{1}}{\left(1+\alpha V_{1}\right)^{2}} \\
\frac{b \beta_{1} V_{1}}{1+\alpha V_{1}}-\sigma b \beta_{1} V_{1} & -\left(\sigma b \beta_{1} V_{1}+\mu_{1}+\gamma\right) & \frac{b \beta_{1} S_{1}}{\left(1+\alpha V_{1}\right)^{2}}+\sigma b \beta_{1}\left(\frac{\Lambda_{1}}{\mu_{1}}-S_{1}-I_{1}\right.
\end{array}\right) \\
& B_{2}=\left(\begin{array}{ccc}
0 & 0 & 0 \\
0 & 0 & 0 \\
0 & b \beta_{2}\left(\frac{\Lambda_{2}}{\mu_{2}}-V_{1}\right) & -b \beta_{2} I_{1}
\end{array}\right) \text {, } \\
& \phi(\theta)=\left(\phi_{1}(\theta) \phi_{2}(\theta) \phi_{3}(\theta)\right)^{\mathrm{T}} \in C, \\
& f_{1}=-\frac{b \beta_{1}}{\left(1+\alpha V_{1}\right)^{2}} \phi_{1}(0) \phi_{3}(0)+\frac{\alpha b \beta_{1}}{\left(1+\alpha V_{1}\right)^{3}} \phi_{1}(0) \phi_{3}^{2}(0)+\frac{\alpha b \beta_{1} S_{1}}{\left(1+\alpha V_{1}\right)^{3}} \phi_{3}^{2}(0) \\
& -\frac{\alpha^{2} b \beta_{1}}{\left(1+\alpha V_{1}\right)^{4}} \phi_{1}(0) \phi_{3}^{3}(0)-\frac{\alpha^{2} b \beta_{1} S_{1}}{\left(1+\alpha V_{1}\right)^{4}} \phi_{3}^{3}(0)+\cdots, \\
& f_{2}=-\sigma b \beta_{1} \phi_{1}(0) \phi_{3}(0)-\sigma b \beta_{1} \phi_{2}(0) \phi_{3}(0)+\frac{b \beta_{1}}{\left(1+\alpha V_{1}\right)^{2}} \phi_{1}(0) \phi_{3}(0)-\frac{\alpha b \beta_{1}}{\left(1+\alpha V_{1}\right)^{3}} \phi_{1}(0) \phi_{3}^{2}(0) \\
& -\frac{\alpha b \beta_{1} S_{1}}{\left(1+\alpha V_{1}\right)^{3}} \phi_{3}^{2}(0)+\frac{\alpha^{2} b \beta_{1}}{\left(1+\alpha V_{1}\right)^{4}} \phi_{1}(0) \phi_{3}^{3}(0)+\frac{\alpha^{2} b \beta_{1} S_{1}}{\left(1+\alpha V_{1}\right)^{4}} \phi_{3}^{3}(0)+\cdots, \\
& f_{3}=-b \beta_{2} \phi_{2}(-1) \phi_{3}(-1) \text {. }
\end{aligned}
$$


Applying the Riesz representation theorem, there exists a $3 \times 3$ matrix-valued function $\eta(\cdot, \mu):[-1,0] \longrightarrow R^{3 \times 3}$, such that $L_{\mu} \phi=\int_{-1}^{0} d \eta(\theta, \mu) \phi(\theta), \phi \in C$. We choose

$$
\eta(\theta, \mu)=(\tilde{\tau}+\mu) B_{1} \delta(\theta)+(\tilde{\tau}+\mu) B_{2} \delta(\theta+1),
$$

where $\delta$ is the Dirac delta function, meeting $\delta(\theta)=0(\theta \neq 0)$ and $\int_{-\infty}^{+\infty} \delta(\theta) d \theta=1$.

We define for $\phi \in C\left([-1,0], R^{3}\right)$,

$$
\begin{aligned}
& A(\mu) \phi= \begin{cases}\frac{d \phi(\theta)}{d \theta}, & \theta \in[-1,0), \\
\int_{-1}^{0} d \eta(s, \mu) \phi(s), & \theta=0,\end{cases} \\
& R(\mu) \phi= \begin{cases}0, & \theta \in[-1,0), \\
f(\mu, \phi), & \theta=0 .\end{cases}
\end{aligned}
$$

Thus, (68) becomes

$$
\dot{x}_{t}=A(\mu) x_{t}+R(\mu) x_{t},
$$

where $x_{t}=x(t+\theta), \theta \in[-1,0]$.

In order to construct coordinates to describe the integral manifold near the origin, we need to define inner product and the adjoint operator $A^{*}=A^{*}(0)$ of $A$ as follows:

$$
\begin{aligned}
A^{*} \psi(s)= \begin{cases}-\frac{d \psi(s)}{d s}, & s \in(0,1], \\
\int_{-1}^{0} d \eta^{\mathrm{T}}(t, 0) \psi(-t), & s=0, \\
\langle\psi, \phi\rangle & =\bar{\psi}^{\mathrm{T}}(0) \phi(0)-\int_{\theta=-1}^{0} \int_{\xi=0}^{\theta} \bar{\psi}^{\mathrm{T}}(\xi-\theta) d \eta(\theta) \phi(\xi) d \xi,\end{cases}
\end{aligned}
$$

where $\psi \in C\left([0,1], R^{3}\right)$ and $\eta(\theta)=\eta(\theta, 0)$. Form the discussion in Section 3, we know that $\pm i \omega \widetilde{\tau}$ are eigenvalues of $A(0)$. Thus they are also eigenvalues of $A^{*}$. Define $q(\theta)=$ $\left(1, q_{1}, q_{2}\right)^{\mathrm{T}} e^{i \theta \omega \tau}$ and $q^{*}(s)=D\left(1, q_{1}^{*}, q_{2}^{*}\right)^{\mathrm{T}} e^{-i s \omega \tilde{\tau}}$ to be the eigenvectors of $A(0)$ and $A^{*}$ corresponding to the eigenvalues $i \omega \tilde{\tau}$ and $-i \omega \tilde{\tau}$, then

$$
\begin{aligned}
A(0) q(\theta) & =i \omega \tilde{\tau} q(\theta), \\
A^{*} q^{*}(s) & =-i \omega \tilde{\tau} q^{*}(s) .
\end{aligned}
$$

We can calculate that

$$
q_{1}=\frac{I_{1}\left(\mu_{2}+b \beta_{2} I_{1} e^{-i \omega \tilde{\tau}}+\omega i\right)\left[b \beta_{1} V_{1}\left(1+\alpha V_{1}\right)+\left(1+\alpha V_{1}\right)^{2} \omega i\right]}{b \beta_{1} S_{1} \mu_{2} V_{1} e^{-i \omega \tilde{\tau}}},
$$

$q_{2}=\left(1+\alpha V_{1}\right) \frac{V_{1}}{S_{1}}+\frac{\left(1+\alpha V_{1}\right)^{2} \omega i}{b \beta_{1} S_{1}}$,

$q_{1}^{*}=\frac{b \beta_{1} V_{1}+\left(\mu_{1}-\omega i\right)\left(1+\alpha V_{1}\right)}{b \beta_{1} V_{1}-\sigma b \beta_{1} V_{1}\left(1+\alpha V_{1}\right)}$,

$$
q_{2}^{*}=\frac{I_{1}\left(\sigma b \beta_{1} V_{1}+\mu_{1}+\gamma-\omega i\right)\left[b \beta_{1} V_{1}+\left(\mu_{1}-\omega i\right)\left(1+\alpha V_{1}\right)\right]}{\mu_{2} V_{1} e^{i \omega \tilde{\tau}}\left[b \beta_{1} V_{1}-\sigma b \beta_{1} V_{1}\left(1+\alpha V_{1}\right)\right]} .
$$

According to (75), we know that

$$
\begin{aligned}
\left\langle q^{*}(s), q(\theta)\right\rangle= & \bar{D}\left(1, \bar{q}_{1}^{*}, \bar{q}_{2}^{*}\right)\left(1, q_{1}, q_{2}\right)^{\mathrm{T}} \\
& -\int_{-1}^{0} \int_{\xi=0}^{\theta} \bar{D}\left(1, \bar{q}_{1}^{*}, \bar{q}_{2}^{*}\right) e^{-i(\xi-\theta) \omega \tilde{\tau}} d \eta(\theta)\left(1, q_{1}, q_{2}\right)^{\mathrm{T}} e^{i \xi \omega \tilde{\tau}} d \xi \\
= & \bar{D}\left\{1+\bar{q}_{1}^{*} q_{1}+\bar{q}_{2}^{*} q_{2}-\int_{-1}^{0}\left(1, \bar{q}_{1}^{*}, \bar{q}_{2}^{*}\right) \theta e^{i \theta \omega \tilde{\tau}} d \eta(\theta)\left(1, q_{1}, q_{2}\right)^{\mathrm{T}}\right\} \\
= & \bar{D}\left\{1+\bar{q}_{1}^{*} q_{1}+\bar{q}_{2}^{*} q_{2}+\tilde{\tau} q_{1} \bar{q}_{2}^{*} b \beta_{2}\left(\frac{\Lambda_{2}}{\mu_{2}}-V_{1}\right) e^{-i \omega \tilde{\tau}}-\tilde{\tau} q_{2} \bar{q}_{2}^{*} b \beta_{2} I_{1} e^{-i \omega \tilde{\tau}}\right\} \\
= & 1,
\end{aligned}
$$


thus

$$
D=\frac{1}{1+q_{1}^{*} \bar{q}_{1}+q_{2}^{*} \bar{q}_{2}+\tilde{\tau} e^{i \omega \tilde{\tau}}\left(\bar{q}_{1} q_{2}^{*} b \beta_{2} \frac{\mu_{2} V_{1}}{I_{1}}-\bar{q}_{2} q_{2}^{*} b \beta_{2} I_{1}\right)} .
$$

Next using the same notation as in [11] and a computation process similar to that in [18], we compute the center manifold $C_{0}$ at $\mu=0$.

Let $x_{t}$ be the solution of (74).

Define

$$
\begin{aligned}
z(t) & =\left\langle q^{*}, x_{t}\right\rangle, \\
W(t, \theta) & =x_{t}(\theta)-2 \operatorname{Re}\{z(t) q(\theta)\} .
\end{aligned}
$$

On the center manifold $C_{0}$, we have

$$
W(t, \theta)=W(z(t), \bar{z}(t), \theta)
$$

where

$$
W(z(t), \bar{z}(t), \theta)=W_{20}(\theta) \frac{z^{2}}{2}+W_{11}(\theta) z \bar{z}+W_{02}(\theta) \frac{\bar{z}^{2}}{2}+\cdots
$$

For the solution $x_{t} \in C_{0}$ with $\mu=0$, we can get

$$
\begin{aligned}
z^{\prime}(t) & =i \omega \tilde{\tau} z+\left\langle\bar{q}^{*}(\theta), f(0, W(z, \bar{z}, \theta)+2 \operatorname{Re}\{z q(\theta)\})\right\rangle \\
& =i \omega \tilde{\tau} z+\bar{q}^{*}(0) f(0, W(z, \bar{z}, \theta)+2 \operatorname{Re}\{z q(0)\}) \\
& =i \omega \tilde{\tau} z+\bar{q}^{*}(0) f_{0}(z, \bar{z}) \\
& \triangleq i \omega \tilde{\tau} z+g(z, \bar{z})
\end{aligned}
$$

where

$$
\begin{aligned}
g(z, \bar{z})= & \bar{q}^{*}(0) f_{0}(z, \bar{z})=g_{20} \frac{z^{2}}{2}+g_{11} z \bar{z}+g_{02} \frac{\bar{z}^{2}}{2} \\
& +g_{21} \frac{z^{2} \bar{z}}{2}+\cdots
\end{aligned}
$$

From (80) and (81), we obtain that

$$
\begin{aligned}
x_{t}(\theta) & =\left(x_{1 t}(\theta), x_{2 t}(\theta), x_{3 t}(\theta)\right)^{\mathrm{T}} \\
& =W(t, \theta)+2 \operatorname{Re}\{z q(\theta)\} \\
& =\left(1, q_{1}, q_{2}\right)^{\mathrm{T}} e^{i \omega \tilde{\tau} \theta} z+\left(1, \bar{q}_{1}, \bar{q}_{2}\right)^{\mathrm{T}} e^{-i \omega \tilde{\tau} \theta} \bar{z}+W_{20}(\theta) \frac{z^{2}}{2}
\end{aligned}
$$$$
+W_{11}(\theta) z \bar{z}+W_{02}(\theta) \frac{\bar{z}^{2}}{2}+\cdots
$$

From (70) and (84),

$$
\begin{aligned}
g(z, \bar{z}) & =\bar{q}^{*}(0) f_{0}(z, \bar{z})=\bar{q}^{*}(0) f\left(0, x_{t}\right) \\
& =\tilde{\tau} \bar{D}\left\{2\left(k_{1}+\bar{q}_{1}^{*} k_{2}+\bar{q}_{2}^{*} k_{3}\right) \frac{z^{2}}{2}+\left(l_{1}+\bar{q}_{1}^{*} l_{2}+\bar{q}_{2}^{*} l_{3}\right) z \bar{z}+2\left(m_{1}+\bar{q}_{1}^{*} m_{2}+\bar{q}_{2}^{*} m_{3}\right) \frac{\bar{z}^{2}}{2}+2\left(n_{1}+\bar{q}_{1}^{*} n_{2}+\bar{q}_{2}^{*} n_{3}\right) \frac{z^{2} \bar{z}}{2}\right\}
\end{aligned}
$$

It follows

$$
\begin{aligned}
& g_{20}=2 \tilde{\tau} \bar{D}\left(k_{1}+\bar{q}_{1}^{*} k_{2}+\bar{q}_{2}^{*} k_{3}\right), \\
& g_{11}=\tilde{\tau} \bar{D}\left(l_{1}+\bar{q}_{1}^{*} l_{2}+\bar{q}_{2}^{*} l_{3}\right), \\
& g_{02}=2 \widetilde{\tau} \bar{D}\left(m_{1}+\bar{q}_{1}^{*} m_{2}+\bar{q}_{2}^{*} m_{3}\right), \\
& g_{21}=2 \tilde{\tau} \bar{D}\left(n_{1}+\bar{q}_{1}^{*} n_{2}+\bar{q}_{2}^{*} n_{3}\right),
\end{aligned}
$$


where

$$
\begin{aligned}
& k_{1}=-\frac{b \beta_{1} q_{2}}{\left(1+\alpha V_{1}\right)^{2}}+\frac{\alpha b \beta_{1} S_{1}}{\left(1+\alpha V_{1}\right)^{3}} q_{2}^{2} \\
& k_{2}=-\sigma b \beta_{1} q_{2}\left(1+q_{1}\right)+\frac{b \beta_{1} q_{2}}{\left(1+\alpha V_{1}\right)^{2}}-\frac{\alpha b \beta_{1} S_{1}}{\left(1+\alpha V_{1}\right)^{3}} q_{2}^{2} \\
& k_{3}=-b \beta_{2} q_{1} q_{2} e^{-2 i \omega \tilde{\tau}} \\
& l_{1}=-\frac{b \beta_{1}}{\left(1+\alpha V_{1}\right)^{2}}\left(\bar{q}_{2}+q_{2}\right)+\frac{2 \alpha b \beta_{1} S_{1}}{\left(1+\alpha V_{1}\right)^{3}} q_{2} \bar{q}_{2} \text {, } \\
& l_{2}=-\sigma b \beta_{1}\left(\bar{q}_{2}+q_{2}+q_{1} \bar{q}_{2}+\bar{q}_{1} q_{2}\right)+\frac{b \beta_{1}}{\left(1+\alpha V_{1}\right)^{2}}\left(\bar{q}_{2}+q_{2}\right)-\frac{2 \alpha b \beta_{1} S_{1}}{\left(1+\alpha V_{1}\right)^{3}} q_{2} \bar{q}_{2}, \\
& l_{3}=-b \beta_{2}\left(q_{1} \bar{q}_{2}+\bar{q}_{1} q_{2}\right) \text {, } \\
& m_{1}=-\frac{b \beta_{1} \bar{q}_{2}}{\left(1+\alpha V_{1}\right)^{2}}+\frac{\alpha b \beta_{1} S_{1}}{\left(1+\alpha V_{1}\right)^{3}} \bar{q}_{2}^{2} \\
& m_{2}=-\sigma b \beta_{1} \bar{q}_{2}\left(1+\bar{q}_{1}\right)+\frac{b \beta_{1} \bar{q}_{2}}{\left(1+\alpha V_{1}\right)^{2}}-\frac{\alpha b \beta_{1} S_{1}}{\left(1+\alpha V_{1}\right)^{3}} \bar{q}_{2}^{2} \\
& m_{3}=-b \beta_{2} \bar{q}_{1} \bar{q}_{2} e^{2 i \omega \tilde{\tau}}, \\
& n_{1}=-\frac{b \beta_{1}}{\left(1+\alpha V_{1}\right)^{2}}\left(\frac{W_{20}^{(1)}(0)}{2} \bar{q}_{2}+\frac{W_{20}^{(3)}(0)}{2}+q_{2} W_{11}^{(1)}(0)+W_{11}^{(3)}(0)\right)+\frac{\alpha b \beta_{1}}{\left(1+\alpha V_{1}\right)^{3}}\left(q_{2}^{2}+2 q_{2} \bar{q}_{2}\right)+\frac{\alpha b \beta_{1} S_{1}}{\left(1+\alpha V_{1}\right)^{3}} \\
& \cdot\left(W_{20}^{(3)}(0) \bar{q}_{2}+2 q_{2} W_{11}^{(3)}(0)\right)-\frac{3 \alpha^{2} b \beta_{1} S_{1}}{\left(1+\alpha V_{1}\right)^{4}} q_{2}^{2} \bar{q}_{2} \\
& n_{2}=-\sigma b \beta_{1}\left(\frac{W_{20}^{(1)}(0)}{2} \bar{q}_{2}+\frac{W_{20}^{(3)}(0)}{2}+q_{2} W_{11}^{(1)}(0)+W_{11}^{(3)}(0)+\frac{W_{20}^{(2)}(0)}{2} \bar{q}_{2}+\frac{W_{20}^{(3)}(0)}{2} \bar{q}_{1}+W_{11}^{(2)}(0) q_{2}\right. \\
& \left.+W_{11}^{(3)}(0) q_{1}\right)+\frac{b \beta_{1}}{\left(1+\alpha V_{1}\right)^{2}} \frac{W_{20}^{(1)}(0)}{2} \bar{q}_{2}+\frac{W_{20}^{(3)}(0)}{2}+q_{2} W_{11}^{(1)}(0)+W_{11}^{(3)}(0)-\frac{\alpha b \beta_{1}}{\left(1+\alpha V_{1}\right)^{3}}\left(q_{2}^{2}+2 q_{2} \bar{q}_{2}\right) \\
& -\frac{\alpha b \beta_{1} S_{1}}{\left(1+\alpha V_{1}\right)^{3}}\left(W_{20}^{(3)}(0) \bar{q}_{2}+2 q_{2} W_{11}^{(3)}(0)\right)+\frac{3 \alpha^{2} b \beta_{1} S_{1}}{\left(1+\alpha V_{1}\right)^{4}} q_{2}^{2} \bar{q}_{2}, \\
& n_{3}=-b \beta_{2}\left(\frac{W_{20}^{(2)}(-1)}{2} \bar{q}_{2} \mathrm{e}^{i \omega \tilde{\tau}}+\frac{W_{20}^{(3)}(-1)}{2} \bar{q}_{1} \mathrm{e}^{i \omega \tilde{\tau}}+W_{11}^{(2)}(-1) q_{2} \mathrm{e}^{-i \omega \tilde{\tau}}+W_{11}^{(3)}(-1) q_{1} \mathrm{e}^{-i \omega \tilde{\tau}}\right) .
\end{aligned}
$$

By using (74) and (80), we can know

$$
\begin{aligned}
W^{\prime} & =x_{t}^{\prime}-z^{\prime} q-\bar{z}^{\prime} \bar{q}= \begin{cases}A W-2 \operatorname{Re}\left\{\bar{q}^{*}(0) f_{0} q(\theta)\right\}, & \theta \in[-1,0), \\
A W-2 \operatorname{Re}\left\{\bar{q}^{*}(0) f_{0} q(\theta)\right\}+f_{0}, & \theta=0,\end{cases} \\
& \triangleq A W+H(z, \bar{z}, \theta),
\end{aligned}
$$

where

$$
H(z, \bar{z}, \theta)=H_{20}(\theta) \frac{z^{2}}{2}+H_{11}(\theta) z \bar{z}+H_{02}(\theta) \frac{\bar{z}^{2}}{2}+\cdots
$$

From (81), (89), and (90), we can get

$$
\left\{\begin{array}{l}
(A-2 i \omega \tilde{\tau}) W_{20}(\theta)=-H_{20}(\theta), \\
A W_{11}(\theta)=-H_{11}(\theta) .
\end{array}\right.
$$

From (89), (91), and the definition of $A$, we can calculate

$$
\begin{aligned}
& W_{20}(\theta)=\frac{i g_{20}}{\omega \tilde{\tau}} q(0) e^{i \omega \tilde{\tau} \theta}+\frac{i \bar{g}_{02}}{3 \omega \tilde{\tau}} \bar{q}(0) e^{-i \omega \tilde{\tau} \theta}+\Gamma_{1} e^{2 i \omega \tilde{\tau} \theta}, \\
& W_{11}(\theta)=-\frac{i g_{11}}{\omega \tilde{\tau}} q(0) e^{i \omega \tilde{\tau} \theta}+\frac{i \bar{g}_{11}}{\omega \tilde{\tau}} \bar{q}(0) e^{-i \omega \tilde{\tau} \theta}+\Gamma_{2},
\end{aligned}
$$

where $\Gamma_{i}=\left(\Gamma_{i}^{(1)}, \Gamma_{i}^{(2)}, \Gamma_{i}^{(3)}\right)^{\mathrm{T}} \in R^{3} \quad(i=1,2)$ are the threedimensional vectors.

Now, we determine $\Gamma_{1}$ and $\Gamma_{2}$. By (91) and the definition of $A$, we have 


$$
\begin{aligned}
& \int_{-1}^{0} d \eta(\theta) W_{20}(\theta)=2 i \omega \widetilde{\tau} W_{20}(\theta)-H_{20}(\theta) \\
& \int_{-1}^{0} d \eta(\theta) W_{11}(\theta)=-H_{11}(\theta)
\end{aligned}
$$

where $\eta(\theta)=\eta(\theta, 0)$. By (89), when $\theta=0$,

$$
\begin{aligned}
H(z, \bar{z}, \theta) & =-\bar{q}^{*}(0) f_{0} q(0)-q^{*}(0) \bar{f}_{0} \bar{q}(0)+f_{0} \\
& =-g(z, \bar{z}) q(0)-\bar{g}(z, \bar{z}) \bar{q}(0)+f_{0} .
\end{aligned}
$$

That leads to

$$
\begin{aligned}
H_{20}(0)= & -g_{20} q(0)-\bar{g}_{02} \bar{q}(0) \\
& +2 \widetilde{\tau}\left(\begin{array}{c}
-\frac{b \beta_{1}}{\left(1+\alpha V_{1}\right)^{2}}+\frac{\alpha b \beta_{1} S_{1}}{\left(1+\alpha V_{1}\right)^{3}} q_{2}^{2} \\
G_{0} \\
-b \beta_{2} q_{1} q_{2} e^{-2 i \omega \tau}
\end{array}\right), \\
H_{11}(0)= & -g_{11} q(0)-\bar{g}_{11} \bar{q}(0) \\
& +2 \widetilde{\tau}\left(\begin{array}{c}
-\frac{b \beta_{1}}{\left(1+\alpha V_{1}\right)^{2}} \operatorname{Re}\left(q_{2}\right)+\frac{\alpha b \beta_{1} S_{1}}{\left(1+\alpha V_{1}\right)^{3}} q_{2} \bar{q}_{2} \\
G_{1} \\
-b \beta_{2} \operatorname{Re}\left(q_{1} \bar{q}_{2}\right)
\end{array}\right),
\end{aligned}
$$

$$
\begin{aligned}
G_{0}= & -\sigma b \beta_{1}\left(q_{2}+q_{1} q_{2}\right)+\frac{b \beta_{1} q_{2}}{\left(1+\alpha V_{1}\right)^{2}}-\frac{\alpha b \beta_{1} S_{1}}{\left(1+\alpha V_{1}\right)^{3}} q_{2}^{2}, \\
G_{1}= & \left(\frac{b \beta_{1}}{\left(1+\alpha V_{1}\right)^{2}}-\sigma b \beta_{1}\right) \operatorname{Re}\left(q_{2}\right)-\sigma b \beta_{1} \operatorname{Re}\left(q_{1} \bar{q}_{2}\right) \\
& -\frac{\alpha b \beta_{1} S_{1}}{\left(1+\alpha V_{1}\right)^{3}} q_{2} \bar{q}_{2} .
\end{aligned}
$$

Since

$$
\begin{gathered}
\left(i \omega \tilde{\tau} I-\int_{-1}^{0} e^{i \theta \omega \tilde{\tau}} d \eta(\theta)\right) q(0)=0 \\
\left(-i \omega \tilde{\tau} I-\int_{-1}^{0} e^{-i \theta \tilde{\tau}} d \eta(\theta)\right) \bar{q}(0)=0
\end{gathered}
$$

we have

$$
\begin{aligned}
& \left(2 i \omega \tilde{\tau} I-\int_{-1}^{0} e^{2 i \theta \omega \tilde{\tau}} d \eta(\theta)\right) E_{1} \\
& =2 \widetilde{\tau}\left(\begin{array}{c}
-\frac{b \beta_{1}}{\left(1+\alpha V_{1}\right)^{2}}+\frac{\alpha b \beta_{1} S_{1}}{\left(1+\alpha V_{1}\right)^{3}} q_{2}^{2} \\
G_{0} \\
-b \beta_{2} q_{1} q_{2} e^{-2 i \omega \tau}
\end{array}\right) .
\end{aligned}
$$

We can calculate

where

$$
\begin{aligned}
& \Gamma_{1}^{(1)}=\frac{2}{\Delta_{1}}\left|\begin{array}{ccc}
-\frac{b \beta_{1}}{\left(1+\alpha V_{1}\right)^{2}}+\frac{\alpha b \beta_{1} S_{1}}{\left(1+\alpha V_{1}\right)^{3}} q_{2}^{2} & 0 & \frac{b \beta_{1} S_{1}}{\left(1+\alpha V_{1}\right)^{2}} \\
G_{0} & 2 i \omega+\sigma b \beta_{1} V_{1}+\mu_{1}+\gamma & -\frac{b \beta_{1} S_{1}}{\left(1+\alpha V_{1}\right)^{2}}+Q_{1} \\
-b \beta_{2} q_{1} q_{2} e^{-2 i \omega \tilde{\tau}} & -b \beta_{2}\left(\frac{\Lambda_{2}}{\mu_{2}}-V_{1}\right) e^{-2 i \omega \tilde{\tau}} & 2 i \omega+\mu_{2}+b \beta_{2} I_{1} e^{-2 i \omega \tilde{\tau}}
\end{array}\right|, \\
& \Gamma_{1}^{(2)}=\frac{2}{\Delta_{1}}\left|\begin{array}{ccc}
2 i \omega+\frac{b \beta_{1} V_{1}}{1+\alpha V_{1}}+\mu_{1} & -\frac{b \beta_{1}}{\left(1+\alpha V_{1}\right)^{2}}+\frac{\alpha b \beta_{1} S_{1}}{\left(1+\alpha V_{1}\right)^{3}} q_{2}^{2} & \frac{b \beta_{1} S_{1}}{\left(1+\alpha V_{1}\right)^{2}} \\
-\frac{b \beta_{1} V_{1}}{1+\alpha V_{1}}+\sigma b \beta_{1} V_{1} & G_{0} & -\frac{b \beta_{1} S_{1}}{\left(1+\alpha V_{1}\right)^{2}}+Q_{1} \\
0 & -b \beta_{2} q_{1} q_{2} e^{-2 i \omega \tau} & 2 i \omega+\mu_{2}+b \beta_{2} I_{1} e^{-2 i \omega \tau}
\end{array}\right| \text {, } \\
& \Gamma_{1}^{(3)}=\frac{2}{\Delta_{1}}\left|\begin{array}{ccc}
2 i \omega+\frac{b \beta_{1} V_{1}}{1+\alpha V_{1}}+\mu_{1} & 0 & -\frac{b \beta_{1}}{\left(1+\alpha V_{1}\right)^{2}}+\frac{\alpha b \beta_{1} S_{1}}{\left(1+\alpha V_{1}\right)^{3}} q_{2}^{2} \\
-\frac{b \beta_{1} V_{1}}{1+\alpha V_{1}}+\sigma b \beta_{1} V_{1} & 2 i \omega+\sigma b \beta_{1} V_{1}+\mu_{1}+\gamma & G_{0} \\
0 & -b \beta_{2}\left(\frac{\Lambda_{2}}{\mu_{2}}-V_{1}\right) e^{-2 i \omega \tau} & -b \beta_{2} q_{1} q_{2} e^{-2 i \omega \tau}
\end{array}\right| \text {, }
\end{aligned}
$$


where

$$
\begin{aligned}
& Q_{1}=-\sigma b \beta_{1}\left(\frac{\Lambda_{1}}{\mu_{1}}-S_{1}-I_{1}\right) \\
& G_{0}=-\sigma b \beta_{1}\left(q_{2}+q_{1} q_{2}\right)+\frac{b \beta_{1} q_{2}}{\left(1+\alpha V_{1}\right)^{2}}-\frac{\alpha b \beta_{1} S_{1}}{\left(1+\alpha V_{1}\right)^{3}} q_{2}^{2} \text {, } \\
& \left.\Delta_{1}=\mid \begin{array}{ccc}
2 i \omega+\frac{b \beta_{1} V_{1}}{1+\alpha V_{1}}+\mu_{1} & 0 & \frac{b \beta_{1} S_{1}}{\left(1+\alpha V_{1}\right)^{2}} \\
-\frac{b \beta_{1} V_{1}}{1+\alpha V_{1}}+\sigma b \beta_{1} V_{1} & 2 i \omega+\sigma b \beta_{1} V_{1}+\mu_{1}+\gamma & -\frac{b \beta_{1} S_{1}}{\left(1+\alpha V_{1}\right)^{2}}-\sigma b \beta_{1}\left(\frac{\Lambda_{1}}{\mu_{1}}-S_{1}-I_{1}\right.
\end{array}\right) .
\end{aligned}
$$

Similarly, we have

$$
\int_{-1}^{0} d \eta(\theta) \Gamma_{2}=-2 \widetilde{\tau}\left(\begin{array}{c}
-\frac{b \beta_{1}}{\left(1+\alpha V_{1}\right)^{2}} \operatorname{Re}\left(q_{2}\right)+\frac{\alpha b \beta_{1} S_{1}}{\left(1+\alpha V_{1}\right)^{3}} q_{2}^{2} \\
G_{1} \\
-b \beta_{2} \operatorname{Re}\left(q_{1} \bar{q}_{2}\right)
\end{array}\right)
$$

That leads to

$$
\Gamma_{2}^{(1)}=\frac{2}{\Delta_{2}}\left|\begin{array}{ccc}
-\frac{b \beta_{1}}{\left(1+\alpha V_{1}\right)^{2}} \operatorname{Re}\left(q_{2}\right)+\frac{\alpha b \beta_{1} S_{1}}{\left(1+\alpha V_{1}\right)^{3}} q_{2}^{2} & 0 & \frac{b \beta_{1} S_{1}}{\left(1+\alpha V_{1}\right)^{2}} \\
\Gamma_{2}^{(2)}=\frac{2}{\Delta_{2}} & \sigma b \beta_{1} V_{1}+\mu_{1}+\gamma & -\frac{b \beta_{1} S_{1}}{\left(1+\alpha V_{1}\right)^{2}}+Q_{1} \\
-\frac{b \beta_{1} V_{1}}{1+\alpha V_{1}}+\sigma b \beta_{1} V_{1} & -b \beta_{2}\left(\frac{\Lambda_{2}}{\mu_{2}}-V_{1}\right) & \mu_{2}+b \beta_{2} I_{1}
\end{array}\right|,
$$


where

$$
\begin{aligned}
& G_{1}=\left(\frac{b \beta_{1}}{\left(1+\alpha V_{1}\right)^{2}}-\sigma b \beta_{1}\right) \operatorname{Re}\left(q_{2}\right)-\sigma b \beta_{1} \operatorname{Re}\left(q_{1} \bar{q}_{2}\right)-\frac{\alpha b \beta_{1} S_{1}}{\left(1+\alpha V_{1}\right)^{3}} q_{2} \bar{q}_{2}, \\
& \Delta_{2}=\left|\begin{array}{ccc}
\frac{b \beta_{1} V_{1}}{1+\alpha V_{1}}+\mu_{1} & 0 & \frac{b \beta_{1} S_{1}}{\left(1+\alpha V_{1}\right)^{2}} \\
-\frac{b \beta_{1} V_{1}}{1+\alpha V_{1}}+\sigma b \beta_{1} V_{1} & \sigma b \beta_{1} V_{1}+\mu_{1}+\gamma & -\frac{b \beta_{1} S_{1}}{\left(1+\alpha V_{1}\right)^{2}}-\sigma b \beta_{1}\left(\frac{\Lambda_{1}}{\mu_{1}}-S_{1}-I_{1}\right)
\end{array}\right| .
\end{aligned}
$$

We can calculate $W_{20}(0)$ and $W_{11}(0)$ from (92) and (93). The above analysis shows that $g_{i j}$ in (84) can be represented by using the parameters in the system (7). Thus, we can get the following equation:

$$
\left\{\begin{array}{l}
c_{1}(0)=\frac{i}{2 \omega \tilde{\tau}}\left(g_{20} g_{11}-2\left|g_{11}\right|^{2}-\frac{\left|g_{02}\right|^{2}}{3}\right)+\frac{g_{21}}{2} \\
\mu_{2}=-\frac{\operatorname{Re}\left\{c_{1}(0)\right\}}{\operatorname{Re}\left\{\lambda^{\prime}(\tilde{\tau})\right\}} \\
\beta_{2}=2 \operatorname{Re}\left\{c_{1}(0)\right\}, \\
T_{2}=-\frac{\operatorname{Im}\left\{c_{1}(0)\right\}+\mu_{2} \operatorname{Im}\left\{\lambda^{\prime}(\tilde{\tau})\right\}}{\omega \widetilde{\tau}}
\end{array}\right.
$$

where the signs of $\mu_{2}$ determine the direction of the Hopf bifurcation of the system (7), the signs of $\beta_{2}$ determine the stability of the bifurcated periodic solution, and $T_{2}$ determine the period of the bifurcation periodic solution. We can summarize the following theorem:

Theorem 5.1. For the model (7), the direction and the stability of the periodic solution of Hopf bifurcation is determined by using (105) when $\tau=\widetilde{\tau}$.

(i) If $\mu_{2}>0$, then the Hopf bifurcation is supercritical and the bifurcation periodic solution exists for $\tau>\tilde{\tau}$ in a $\tilde{\tau}$ neighborhood; if $\mu_{2}<0$, the Hopf bifurcation is subcritical and the bifurcation periodic solution exists for $\tau<\tilde{\tau}$ in a $\tilde{\tau}$ neighborhood.

(ii) If $\beta_{2}<0$, then the bifurcation periodic solution is stable; if $\beta_{2}>0$, the bifurcation periodic solution is unstable. (iii) If $T_{2}>0$, then the period of the bifurcation periodic solution increases; if $T_{2}<0$, the period decreases.

\section{Numerical Simulations}

In this section, we use numerical simulations to illustrate our result about the existence of Hopf bifurcation.

The following parameters are selected: $\Lambda_{1}=8, b=0.29$, $\beta_{1}=0.0033, \quad \alpha=0.06, \quad \mu_{1}=0.0029, \quad \sigma=0.48, \quad \gamma=0.56$, $\Lambda_{2}=9, \beta_{2}=0.0059$, and $\mu_{2}=0.03$. We can calculate that $R_{0}=80.2459$ and $E_{1}=(444.9472,450.8920,288.7707)$.

Equation (37) has a positive root $x=0.5779$. We have $\tau^{*}=2.1386$. From Theorem 3.4, we know that the endemic equilibrium $E_{1}$ is locally asymptotically stable for $0<\tau<\tau^{*}$ (Figure 1), and system (7) undergoes a Hopf bifurcation at $E_{1}$ when $\tau=\tau^{*}$. At this time, we can calculate $c_{1}(0)=-2.3788-0.8427 i, \mu_{2}=9.8850>0, \beta_{2}=-4.7576<0$, and $T_{2}=1.7152>0$. According to Theorem 5.1, system (7) can produce a stable periodic solution, the Hopf bifurcation is supercritical at $\tau^{*}$, and the period of the bifurcation periodic solution increases (Figure 2).

\section{Conclusions}

In this paper, we discuss the dynamics of the vector-borne disease model with delay-saturated infection rate and reinfection. By calculation, we have the basic reproductive number $R_{0}$. Through $R_{0}$, we determined the existence of disease-free equilibrium $E_{0}$ and the endemic equilibrium $E_{1}$. According to the characteristic equation of the equilibrium points and using the Routh-Hurwitz criterion, we obtained that if $R_{0}<1$ the disease-free equilibrium will be stable, and the endemic equilibrium is locally asymptotically stable if $R_{0}>1$ and in the absence of time delay. Furthermore, by the fluctuation lemma and the limit theory, we analyzed the global stability of the disease-free equilibrium. We find that the time delay does not affect the 


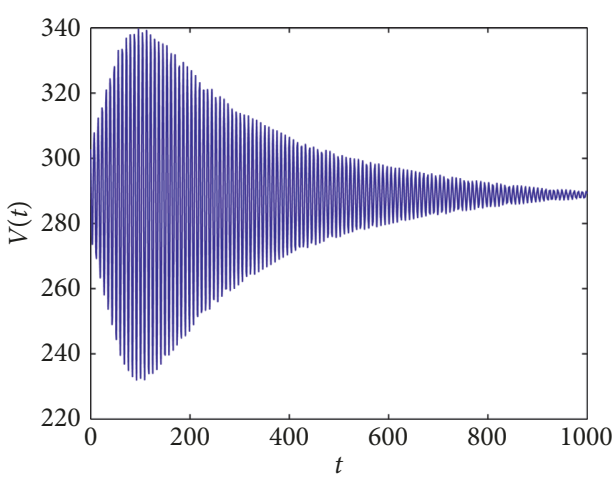

(a)

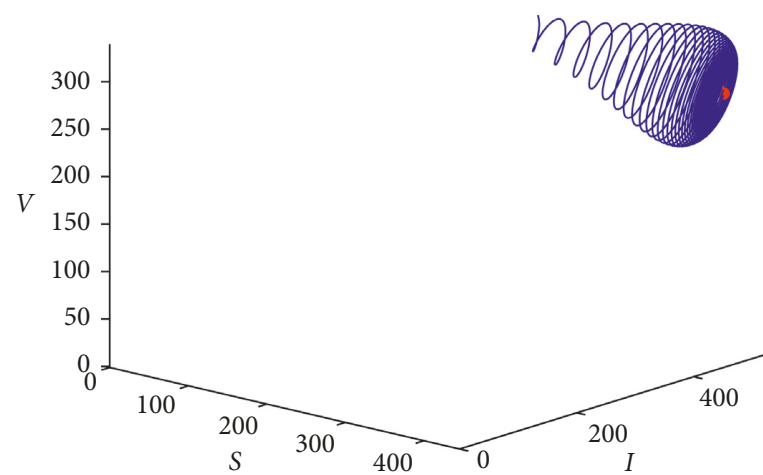

(b)

Figure 1: $E_{1}$ is stable when $\tau=2.12$.

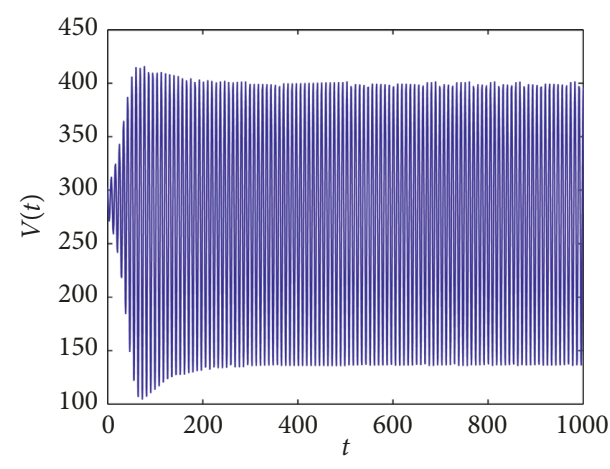

(a)

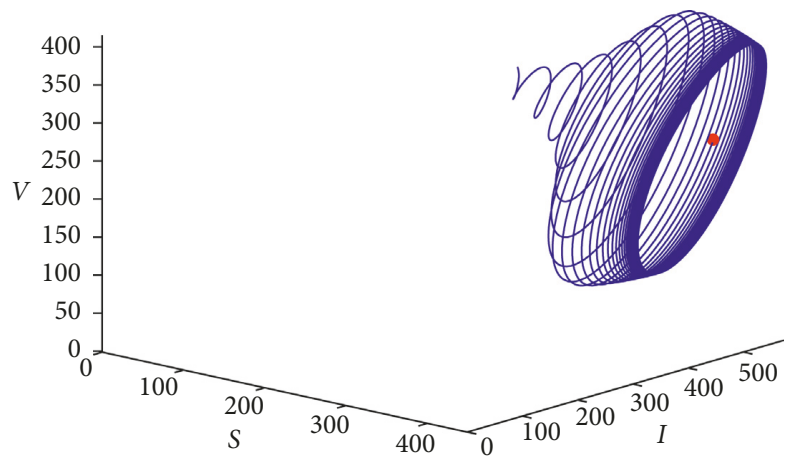

(b)

Figure 2: $E_{1}$ undergoes Hopf bifurcation when $\tau=2.33$.

stability of the boundary equilibrium but can change the stability of $E_{1}$ and lead to the occurrence of Hopf bifurcation. Then by using the Nyquist criterion, we get the maximum length of delay to preserve stability. Next, we found that the conditions for determining the direction and stability of bifurcating periodic solutions. Finally, the correctness of the main conclusion is verified by numerical simulation.

\section{Data Availability}

The data we selected is only to verify the correctness of the results. These data are not real data.

\section{Conflicts of Interest}

The authors declare that they have no conflicts of interest.

\section{Acknowledgments}

This work was supported by the National Natural Science Foundation of China (11471034).

\section{References}

[1] J. Sachs and P. Malaney, "The economic and social burden of malaria," Nature, vol. 415, no. 6872, pp. 680-685, 2002.

[2] A. M. Niger and A. B. Gumel, "Mathematical analysis of the role of repeated exposure on malaria transmission dynamics," Differential Equations and Dynamical Systems, vol. 16, no. 3, pp. 251-287, 2008.

[3] K. Dietz, L. Molineaux, and A. Thomas, "A malaria model tested in the African savannah," Bull World Health Organ, vol. 50, no. 3-4, pp. 347-357, 1974.

[4] C. C. Enweronu-Laryea, G. O. Adjei, B. Mensah, N. Duah, and N. B. Quashie, "Prevalence of congenital malaria in high-risk ghanaian newborns: a cross-sectional study," Malaria Journal, vol. 12, no. 1, pp. 1-5, 2013.

[5] L. Hviid, "Naturally acquired immunity to Plasmodium falciparum in africa," Acta Tropica, vol. 95, no. 3, pp. 265-269, 2005.

[6] L. Cai and X. Li, "Analysis of a simple vector-host epidemic model with direct transmission," Discrete Dynamics in Nature and Society, vol. 2010, Article ID 679613, 12 pages, 2010.

[7] M. Jovanovic and M. Krstic, "Stochastically perturbed vectorborne disease models with direct transmission," Applied Mathematical Modelling, vol. 36, no. 11, pp. 5214-5228, 2012.

[8] H. Wan and J. A. Cui, "A malaria model with two delays," Discrete Dynamics in Nature and Society, vol. 2013, Article ID 601265, 8 pages, 2013.

[9] H.-M. Wei, X.-Z. Li, and M. Martcheva, "An epidemic model of a vector-borne disease with direct transmission and time delay," Journal of Mathematical Analysis and Applications, vol. 342, no. 2, pp. 895-908, 2008.

[10] L. M. Cai, M. Martcheva, and X. Z. Li, "Competitive exclusion in a vector-host epidemic model with distributed delay," Journal of Biological Dynamics, vol. 7, no. 1, pp. 1-21, 2013.

[11] B. D. Hassard, N. D. Kazariniff, and Y. H. Wan, "Theory and application of Hopf bifurcation," London Mathematical Society Lecture Note Series, vol. 41, 1981. 
[12] J. Hale, "Theory and application of Hopf bifurcation," in London Math Society Lecture Note Series, Cambridge University Press, Cambridge, UK, 1977.

[13] C. Castillochavez and H. R. Thieme, "Asymptotically autonomous epidemic models," in Mathematical Population Dynamics: Analysis of Heterogeneity, Theory of Epidemics, Vol. 1, Wuerz, Winnipeg, Canada, 1994.

[14] J. Xu and Y. Zhou, "Hopf bifurcation and its stability for a vector-borne disease model with delay and reinfection," Applied Mathematical Modelling, vol. 40, no. 3, pp. 1685-1702, 2016.

[15] W. M. Hirsch, H. Hanisch, and J.-P. Gabriel, "Differential equation models of some parasitic infections: methods for the study of asymptotic behavior," Communications on Pure and Applied Mathematics, vol. 38, no. 6, pp. 733-753, 1985.

[16] Y. Song and S. Yuan, "Bifurcation analysis in a predator-prey system with time delay," Nonlinear Analysis: Real World Applications, vol. 7, no. 2, pp. 265-284, 2006.

[17] H. L. Freedman and V. Sree Hari Rao, "The trade-off between mutual interference and time lags in predator-prey systems," Bulletin of Mathematical Biology, vol. 45, no. 6, pp. 991-1004, 1983.

[18] X.-Y. Meng, H.-F. Huo, X.-B. Zhang, and H. Xiang, "Stability and Hopf bifurcation in a three-species system with feedback delays," Nonlinear Dynamics, vol. 64, no. 4, pp. 349-364, 2011. 


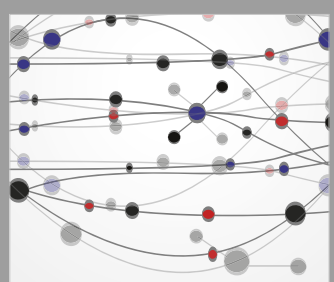

The Scientific World Journal
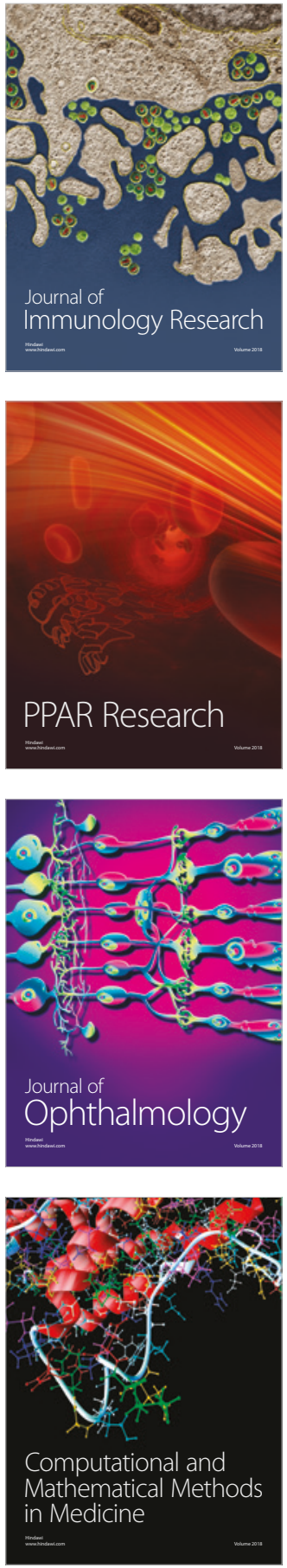

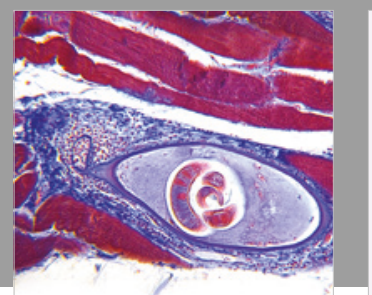

Gastroenterology Research and Practice

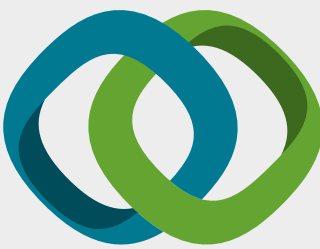

\section{Hindawi}

Submit your manuscripts at

www.hindawi.com
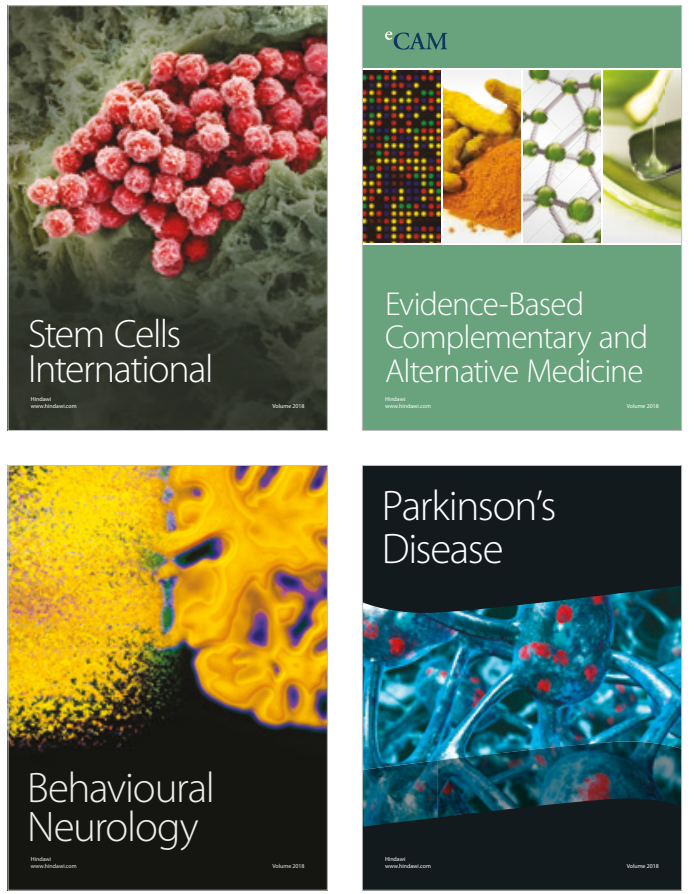

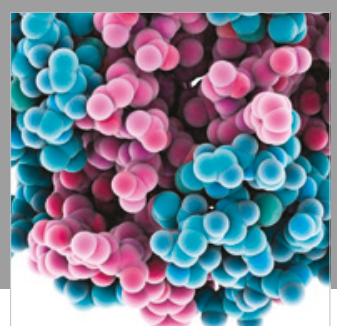

ournal of

Diabetes Research

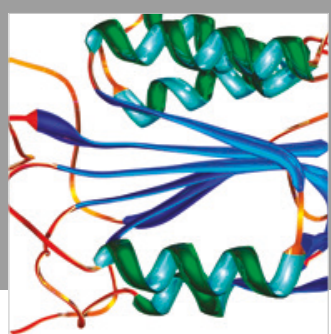

Disease Markers
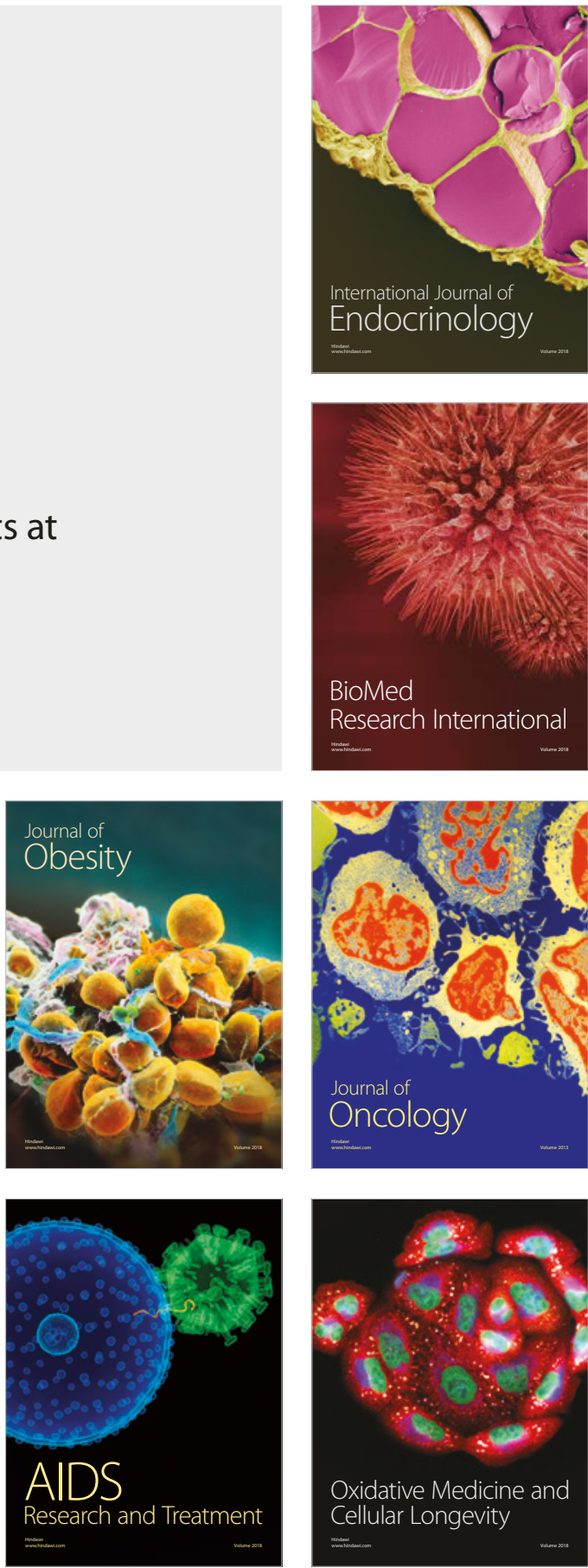